\title{
Resonant Machine Learning Based on Complex Growth Transform Dynamical Systems
}

\author{
Oindrila Chatterjee, Student Member, IEEE, and Shantanu Chakrabartty, Senior Member, IEEE
}

\begin{abstract}
Traditional energy-based learning models associate a single energy metric to each configuration of variables involved in the underlying optimization process. Such models associate the lowest energy state to the optimal configuration of variables under consideration, and are thus inherently dissipative. In this paper we propose an energy-efficient learning framework that exploits structural and functional similarities between a machine learning network and a general electrical network satisfying the Tellegen's theorem. In contrast to the standard energybased models, the proposed formulation associates two energy components, namely, active and reactive energy to the network. This ensures that the network's active-power is dissipated only during the process of learning, whereas the reactive-power is maintained to be zero at all times. As a result, in steady-state, the learned parameters are stored and self-sustained by electrical resonance determined by the network's nodal inductances and capacitances. Based on this approach, this paper introduces three novel concepts: (a) A learning framework where the network's active-power dissipation is used as a regularization for a learning objective function that is subjected to zero total reactive-power constraint; (b) A dynamical system based on complex-domain, continuous-time growth transforms which optimizes the learning objective function and drives the network towards electrical resonance under steady-state operation; and (c) An annealing procedure that controls the trade-off between active-power dissipation and the speed of convergence. As a representative example, we show how the proposed framework can be used for designing resonant support vector machines (SVMs), where we show that the support-vectors correspond to an LC network with self-sustained oscillations. We also show that this resonant network dissipates less active-power compared to its non-resonant counterpart.
\end{abstract}

Index Terms-Electrical Resonance, Tellegen's theorem, Energy-efficient learning models, Energy-based learning models, Complex domain machine learning, Support Vector Machines, Resonant Networks, Coupled Oscillators.

\section{INTRODUCTION}

$\mathbf{F}$ ROM an energy point of view, the dynamics of a machine learning framework is analogous to an electrical network since both the networks evolve over a conservation manifold to attain a low-energy state. In literature, this analogy has served as the basis for energy-based learning models, where the learning objective function is mapped to an equivalent network energy. [1], [2]. The network variables then evolve according to some physical principles subject to network constraints to seek out an energy optimal state. However,

Both the authors are with the Department of Electrical and Systems Engineering, Washington University in St. Louis, St. Louis, Missouri 63130 , USA. All correspondences regarding this manuscript should be addressed to shantanu@wustl.edu.

This work was supported in part by a research grant from the National Science Foundation (ECCS:1550096).

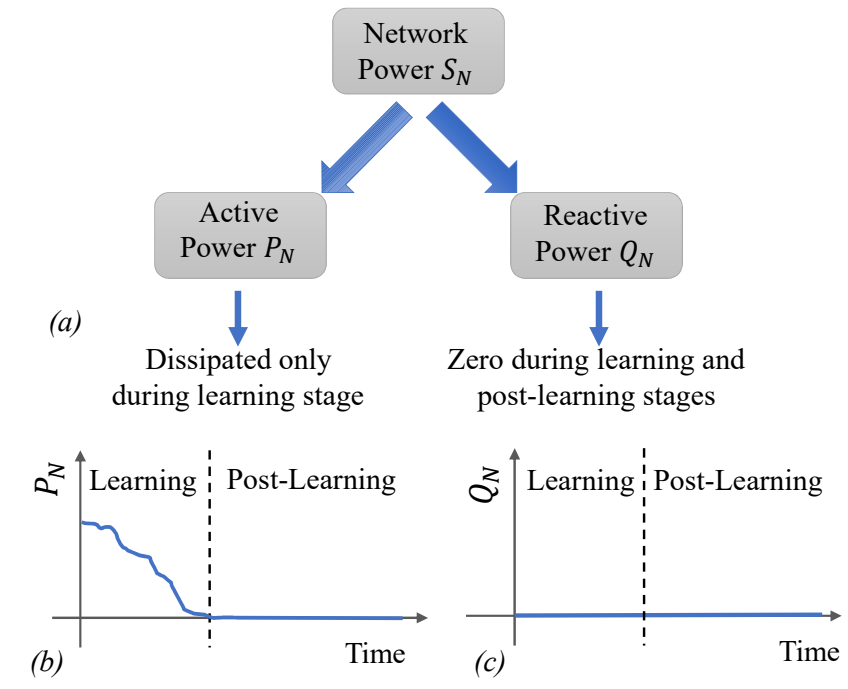

Figure 1. (a) The total or apparent power $S_{N}$ of the electrical network comprising of the active-power $P_{N}$ or the dissipated power and the reactivepower $Q_{N}$ or the power used for energy-storage. The goal of the proposed resonant learning framework: (b) minimizing active-power $P_{N}$ during learning and ensuring $P_{N}=0$, post-learning or steady-state; and (c) maintaining $Q_{N}=0$ in learning and post-learning phases.

even if the solution reached by such energy-based learning models may be optimal from the objective function point of view, it may not be the most energy-efficient solution when considering an actual physical implementation. This is because most of these formulations assume that the energy being minimized in the network is dissipative in nature. Whereas, in a physical electrical network, the total power $S_{N}$ (also known as the apparent power) comprises not only of the dissipative component (also referred to as the active-power) but also a latent or stored non-dissipative component (also referred to as the reactive-power) [3], [4]. This is illustrated in Figure [1,a) and can be mathematically expressed as:

$$
\begin{array}{r}
\text { Total Network Power } S_{N}=\text { Active Power } P_{N} \\
+j \times \text { Reactive Power } Q_{N}
\end{array}
$$

where $j=\sqrt{-1}$ denotes the imaginary component. While the active-power $P_{N}$ represents the rate-of-energy loss in the network, the reactive-power $Q_{N}$ represents the rate of change in stored energy in the network's electric and magnetic fields (typically modeled as lumped capacitive and inductive elements). In the design of electrical networks, reactive-power is generally considered to be a nuisance since it represents the latent power that does not perform any useful work [5], [6]. However, from the point of view of learning, the reactivepower could be useful not only for storing the learned param- 
eters of the network, but could also improve the dynamics of the network during learning. In this paper, we propose such a framework which exploits both active and reactive network power for learning and memory. The objective will be to achieve a network power profile as illustrated in Figures 1 (b) and (c). During learning, the network will optimize its active-power $\left(P_{N}\right)$ and under steady-state condition or postlearning, ensure $P_{N}=0$. The reactive-power $\left(Q_{N}\right)$, on the other hand, will always be maintained at zero. This implies that the stored energy (mathematically - the time-integral of the reactive-power) is conserved across the learning and postlearning phases respectively. Thus, during the post-learning phase or in steady-state, the network will not dissipate any power, and the reactive energy is used to maintain its current network state or memory.

This steady-state condition corresponds to a state of electrical resonance, and in Section III we generalize this concept to a framework of resonant machine learning. To reach this steady-state, in Section IV, we present a dynamical system based on complex-domain continuous-time growth-transforms, which extends our previous work on growth transform networks using real-variables [7]. The complex-domain formulation allows manipulation of the relative phase between the voltage and current variables associated with the network nodes and thus is used to optimize the active-power dissipation during learning. While the approach could be applied to different learning networks, in Section V, we use this framework for designing resonant one-class support vector machines (SVMs) [8]. In this context, we also compare the performance of the resonant SVM model with its non-resonant variant on both synthetic and real datasets. Finally, Section VI concludes the paper with a brief discussion on the implication of the proposed model when applied to other network-based models that do not involve learning, for instance, the coupled oscillator networks [9].

The key contributions of the paper can be summarized as follows:

- Contrary to standard energy-based models which optimize for a single energy metric, we map a learning problem to an electrical network with two energy components: dissipative(active) and non-dissipative(reactive) energy.

- Active power dissipation is introduced as a regularizer in the original learning problem while enforcing zero total reactive power by driving the network into electrical resonance. This ensures zero active power dissipation in the post-learning stage.

- We present a novel growth transform-based complex dynamical system for optimizing the cost function that ensures a resonant condition in steady-state.

- We propose an annealing schedule that can trade-off speed of convergence with active-power dissipation for different applications.

\section{A. Notations}

For the rest of the paper, we will follow the mathematical notations summarized in Table 1 below:
Table 1: Notations

\begin{tabular}{|c|l|}
\hline Variable & Definition \\
\hline $\mathbb{R}_{+}$ & One-dimensional positive real vector space \\
\hline $\mathbb{R}^{N}$ & N-dimensional real vector space \\
\hline $\mathbb{C}$ & One-dimensional complex vector space \\
\hline$|z|$ & magnitude of a complex variable $z$ \\
\hline $\operatorname{Re}(z)$ & real part of a complex variable $z$ \\
\hline $\operatorname{Im}(z)$ & imaginary part of a complex variable $z$ \\
\hline$z^{*}$ & complex conjugate of a complex variable $z$ \\
\hline$z(t)$ & a continuous-time complex variable at time $t$ \\
\hline$z_{n}$ & a discrete-time complex variable at the $n^{\text {th }}$ time step \\
\hline
\end{tabular}

\section{BACKGROUND AND RELATED WORKS}

\section{A. Energy-based Learning Models}

The principle of minimum energy states that all selforganizing physical systems evolve dynamically over an intrinsic conservation manifold to attain their minimum energy configuration in the steady state [10]. Energy-based machine learning models follow a similar cue, where the goal is to find the optimal configuration of a predetermined energy landscape determined by the learning problem at hand [1], [11]. Some of the most notable examples of energy-based models are those based on the Ising model of statistical mechanics, like the Hopfield network [2], and its stochastic counterpart the Boltzmann machine [12] and its variants [13]. In these models, the network converges to the local minimum of a Lyapunov function in steady state. Another class of energy-based models (EBMs) [1] propose a unified learning framework by mapping the learning problem to a global scalar "energy function". The algorithm minimizes a loss functional to seek out an optimal energy landscape that associates lowest energy to the observed configuration of variables involved in the underlying optimization process. These methods are essentially non-probabilistic, and often involve intractable integrals which require MCMC methods for normalization when applied to probabilistic frameworks. Different classes of optimization algorithms like contrastive divergence, contrastive hebbian learning, equilibrium propagation etc. [14] have been proposed for a variety of supervised, unsupervised and reinforcement learning applications [15]-[18] in the context of energybased models. However, all of these approaches consider an energy metric that solely depends on the learning task under consideration. This implies that in an analog implementation of the model, the optimal solution may not be the most energyefficient one.

\section{B. Complex-domain Machine Learning Models}

A variety of complex domain learning models have been proposed in literature for different learning algorithms, e.g., complex neural networks, complex support vector machines, complex deep networks etc [19]-[23]. In addition to providing 

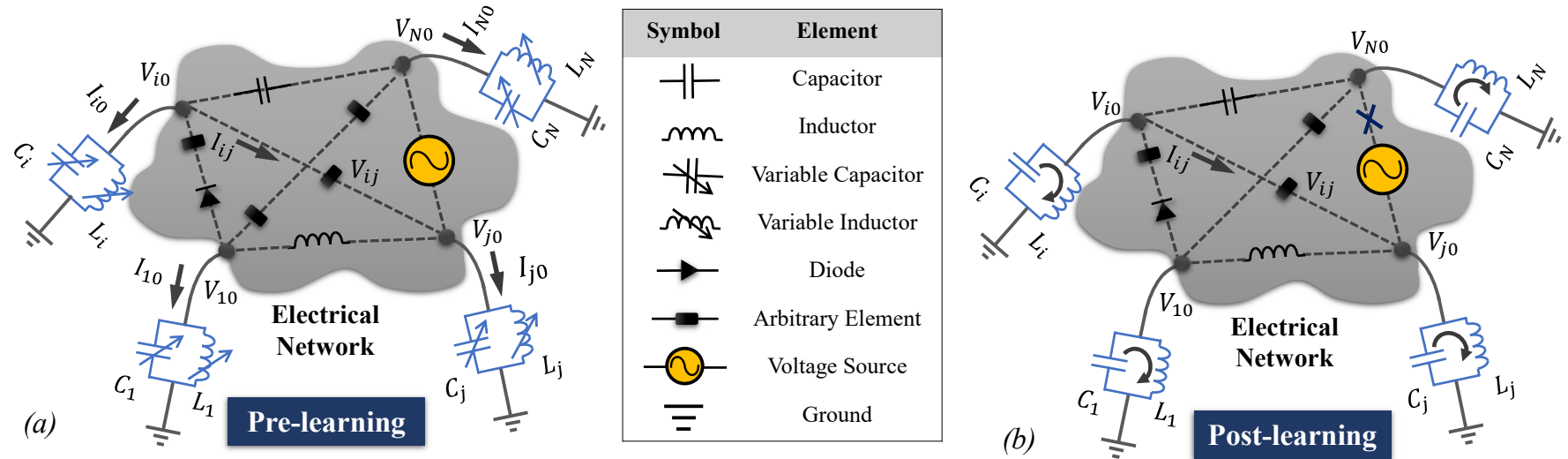

Figure 2. Equivalent network model comprising of $N$ electrical nodes, with an inductance and capacitance element associated with each of the nodes. (a) Learning is equivalent to changing the values of inductive and capacitive elements; and (b) in steady-state the network is driven into electrical resonance.

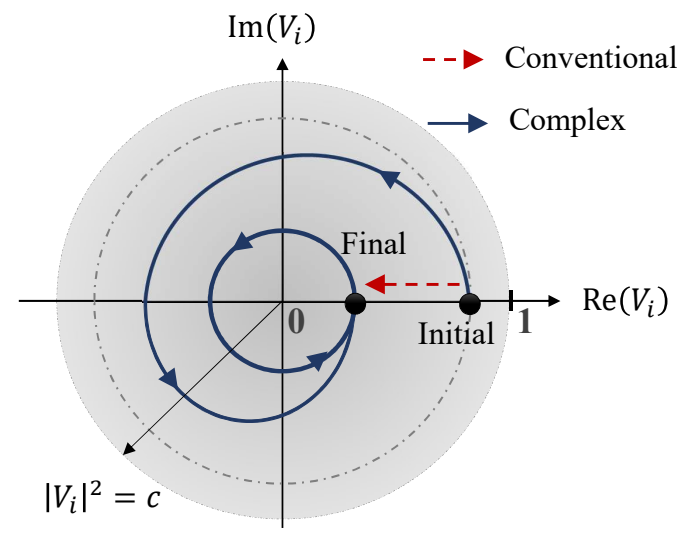

Figure 3. Illustration showing that operating in the complex domain allows different possible learning trajectories from an initial state to the final steadystate. Regularization with respect to the phase-factor could then be used to select the trajectory with an optimal active-power dissipation profile, and results in limit cycle oscillations in steady-state. The circles indicate the constant magnitude loci.

the phase variables which allow for another degree of freedom, other advantages of the complex domain operation have been shown in these works. It has been demonstrated that complex learning models lead to a richer set of dynamics, noise robustness and better convergence properties in the case of classification problems [20], [24], [25]. Moreover, phase information might provide additional insights in the context of many complex-valued physical signals (or complex domain transforms of physical signals, e.g., Fourier or wavelet transforms) [20], [26]. However, most of these complex learning models either treat the real and imaginary components of the signal separately, or do not utilize the entire phase space when operating in the complex domain.

\section{Optimization And Electrical ResonanCE}

Consider an electrical network as shown in Figure 2(a), comprising of $N$ internal nodes. The voltage difference between the $i^{\text {th }}$ and $j^{\text {th }}$ nodes is denoted by $V_{i j}$, with $V_{i 0}$ being the $i^{\text {th }}$ nodal voltage with respect to ground terminal (referred to as the $0^{\text {th }}$ node). Similarly, the current flowing between the $i^{\text {th }}$ and $j^{\text {th }}$ nodes is given by $I_{i j}$, and $I_{i 0}$ denotes the current flowing out of the $i^{\text {th }}$ node into the ground terminal. Then, according to the Tellegen's theorem [27]:

$$
\sum_{i} V_{i j} I_{i j}^{*}=0,
$$

which states that the total complex electrical power or apparent power is zero. Isolating the apparent power flowing from the nodes to the ground terminal from that flowing between the internal nodes, we have:

$$
\begin{gathered}
\sum_{i \neq j, 0 ; j \neq 0}\left(V_{i j}\right) I_{i j}^{*}+\sum_{i}\left(V_{i 0}\right) I_{i 0}^{*}=0 \\
\Longrightarrow \sum_{i=1}^{N} V_{i 0} I_{i 0}^{*}=-\sum_{i, j=1}^{N} V_{i j} I_{i j}^{*} \\
\Longrightarrow S_{T}=-S_{N} \\
\Longrightarrow P_{T}+j Q_{T}=-P_{N}-j Q_{N} \\
\Longrightarrow\left|P_{T}\right|=\left|P_{N}\right| \\
\Longrightarrow\left|Q_{T}\right|=\left|Q_{N}\right|
\end{gathered}
$$

where $S_{T}=\sum_{i} V_{i 0} I_{i 0}^{*}$ is the nodal apparent power, and $P_{T}=\sum_{i} \operatorname{Re}\left\{V_{i 0} I_{i 0}^{*}\right\}$ and $Q_{T}=\sum_{i} \operatorname{Im}\left\{V_{i 0} I_{i 0}^{*}\right\}$ are the total active and reactive power consumed at the nodes. Similarly, $S_{N}, P_{N}$ and $Q_{N}$ represent the apparent, active and reactive power consumed due to current flow between the network nodes (other than the ground terminal). Note that this result holds even if active-power sources are embedded in the network, as shown in Figure 2 (a). Thus, Equations (3) imply that if we minimize the active-power at the nodes of the network $P_{T}$ subject to the constraint that the nodal reactive power $Q_{T}=0$, then the formulation would be equivalent to minimizing the network active-power $P_{N}$ while ensuring that the network reactive power $Q_{N}=0$. This result can be expressed as:

$$
\begin{aligned}
\min _{\left\{V_{i}, I_{i} \in \mathbb{C}\right\}} & \mathcal{D}=\sum_{i=1}^{N}\left|\operatorname{Re}\left\{V_{i} I_{i}^{*}\right\}\right|^{2} \\
\text { s.t. } & \sum_{i} \operatorname{Im}\left\{V_{i} I_{i}^{*}\right\}=0
\end{aligned}
$$


where we have used the notations $V_{i 0}=V_{i}$ and $I_{i 0}=I_{i}$ for the sake of brevity. If we assume that the $i^{\text {th }}$ node is associated with a lumped capacitance $C_{i}$, and a lumped inductance $L_{i}$, ensuring zero nodal reactive-power implies

$$
\sum_{i=1}^{N} V_{i}\left(C_{i} \frac{d V_{i}}{d t}\right)^{*}+\left(L_{i} \frac{d I_{i}}{d t}\right) I_{i}^{*}=0
$$

where $\left(C_{i} \frac{d V_{i}}{d t}\right)$ and $\left(L_{i} \frac{d I_{i}}{d t}\right)$ represent the current flowing through $C_{i}$ and the voltage across $L_{i}$ respectively. Equation (5) is equivalent to:

$$
\sum_{i=1}^{N}\left(\frac{1}{2} C_{i}\left|V_{i}\right|^{2}+\frac{1}{2} L_{i}\left|I_{i}\right|^{2}\right)=E_{0}
$$

where $\left|V_{i}\right|^{2}=V_{i} V_{i}^{*}$ and $\left|I_{i}\right|^{2}=I_{i} I_{i}^{*}$, implying that the total network reactive energy is conserved to be equal to some constant value $E_{0}$. Satisfying this constraint is equivalent to sustaining a condition of electrical resonance. The optimization problem (4) can be transformed as:

$$
\begin{array}{r}
\min _{\left\{\left|V_{i}\right|,\left|I_{i}\right| \in \mathbb{R}_{+}, \phi_{i} \in \mathbb{R}\right\}} \mathcal{D}=\sum_{i=1}^{N}\left|V_{i}\right|^{2}\left|I_{i}\right|^{2} \cos ^{2} \phi_{i} \\
\text { s.t. } \quad \sum_{i=1}^{N}\left(\frac{1}{2} C_{i}\left|V_{i}\right|^{2}+\frac{1}{2} L_{i}\left|I_{i}\right|^{2}\right)=E_{0}, \quad\left|\phi_{i}\right| \leq \pi \forall i
\end{array}
$$

where $\phi_{i}$ denotes the phase-angle between the voltage and current phasors at the $i^{\text {th }}$ node. Note that the optimization in Equation (7) admits only three types of solutions in steadystate: (a) $\left(\left|I_{i}\right| \neq 0,\left|V_{i}\right| \neq 0,\left|\phi_{i}\right|=\pi / 2\right)$ which corresponds to a resonant LC tank; (b) $\left(\left|I_{i}\right|=0,\left|V_{i}\right| \neq 0\right)$ which corresponds to an electrically isolated or floating node; and (c) $\left(\left|I_{i}\right| \neq 0,\left|V_{i}\right|=0\right)$ which corresponds to a short-circuit. In Appendix A we illustrate steady-state resonance conditions using a simple LC tank. Note that in all cases, active power is dissipated only during the learning phase, where $C_{i}$ and $L_{i}$ adapt to change the relative magnitude of the voltage and current variables. Figure 2(b) illustrates this resonant condition of the network in the post-learning stage, whereby active power sources in the network get electrically isolated from the rest of the network. The lumped capacitance and inductance associated with the LC network at the terminal nodes adapt such that the resonant frequency condition in maintained in steady-state (see Appendix A). This implies that in steadystate, the learned network parameters are stored and sustained by the resonant electric and magnetic fields of the LC tanks.

The constraint in Equation (8) can be simplified by normalizing with respect to $E_{0}$ such that:

$$
\sum_{i=1}^{N}\left(\left|V_{i}\right|^{2}+\left|I_{i}\right|^{2}\right)=1
$$

where $V_{i} \leftarrow \sqrt{\frac{C_{i}}{2 E_{0}}} V_{i}$ and $I_{i} \leftarrow \sqrt{\frac{L_{i}}{2 E_{0}}} I_{i}$ represent the dimension-less voltages and currents. Henceforth, unless stated otherwise, we will use dimension-less quantities in our derivations.
We now extend the optimization framework in Equation 7 to include a general optimization function $\mathcal{H}$ as:

$$
\begin{aligned}
\min _{\left\{\left|V_{i}\right|,\left|I_{i}\right|, \phi_{i}\right\}} & \mathcal{L}\left(\left\{\left|V_{i}\right|,\left|I_{i}\right|, \phi_{i}\right\}\right)=\mathcal{H}\left(\left\{\left|V_{i}\right|,\left|I_{i}\right|\right\}\right)+\beta \mathcal{D} \\
\text { s.t. } & \sum_{i=1}^{N}\left(\left|V_{i}\right|^{2}+\left|I_{i}\right|^{2}\right)=1,\left|\phi_{i}\right| \leq \pi \forall i,
\end{aligned}
$$

In this formulation, the active-power dissipation $\mathcal{D}$ in Equation (10) acts as a regularization function with $\beta \geq 0$ being a hyper-parameter. Note that the objective function $\mathcal{H}\left(\left\{\left|V_{i}\right|,\left|I_{i}\right|\right\}\right)$ is only a function of the magnitudes of the voltages and currents and is independent of the phase-angle $\phi_{i}$. This ensures independent control of the magnitudes and the phase to achieve the desired objective of optimizing the active-power dissipation. This is illustrated in Figure 3 where controlling the phase allows different paths from the initial to the final state, whereas evolution over the realdomain allows only one possible path. The complex-domain approach thus results in steady-state limit cycle oscillations that encode the final solution. Compared to other complexdomain machine learning frameworks [20], [23], the proposed formulation avoids non-linear processing of phase/frequency which produces unwanted higher-order harmonics. This would have made it difficult to maintain the network in the state of resonance (at specific set of frequencies) under steady-state.

The two important properties of the optimization framework in Equation (10) are as follows:

- For a convex cost function $H$, we have

$$
\min _{\left\{\left|V_{i}\right|,\left|I_{i}\right|, \phi_{i}\right\}} \mathcal{L}\left(\left\{\left|V_{i}\right|,\left|I_{i}\right|, \phi_{i}\right\}\right)=\min _{\left\{\left|V_{i}\right|,\left|I_{i}\right|\right\}} \mathcal{H}\left(\left\{\left|V_{i}\right|,\left|I_{i}\right|\right\}\right)
$$

This result follows from the three possible solutions of optimization problem (7).

- If $\beta$ is slowly annealed to a sufficiently high value, then $\phi_{i} \rightarrow \pi / 2$ under steady state for $i:\left|V_{i}\right|\left|I_{i}\right| \neq 0$. This implies that network achieves zero active power dissipation in steady state. Note that the method also holds for non-convex objective functions as well. In this case however, the network might show resonant behavior at a locally optimal solution.

Example 1: Consider a single-variable quadratic optimization problem of the form $\mathcal{H}_{1}(x)=x^{2}$, subject to the constraint $|x| \leq 1, x \in \mathbb{R}$. Substituting $x=|V|^{2}-|I|^{2}$, the problem can be mapped (please see Appendix B for more details) into the form equivalent to Equation $[10$ as:

$$
\begin{array}{ll}
\min _{\{|V|,|I|, \phi\}} & \mathcal{L}_{1}=\left(|V|^{2}-|I|^{2}\right)^{2}+\beta|V|^{2}|I|^{2} \cos ^{2} \phi \\
& \text { s.t. }|V|^{2}+|I|^{2}=1, \quad|\phi| \leq \pi
\end{array}
$$

Figures 4(a)-(c) plots $\mathcal{L}_{1}$ for different values of $\beta$. As shown in 4(a), and as expected for $\beta=0$, the cost function has several minima (or attractors), whereas for $\beta>0$, the minima corresponds to $\phi= \pm \pi / 2$, for which the active-power dissipation is zero. The Figures 4(b)-(c) show that controlling $\beta$ will control the optimization landscape (without changing the location of the attractors) and will determine the attractor trajectory. This feature has been exploited in later sections to optimize the active-power dissipation profile during the learning phase. 

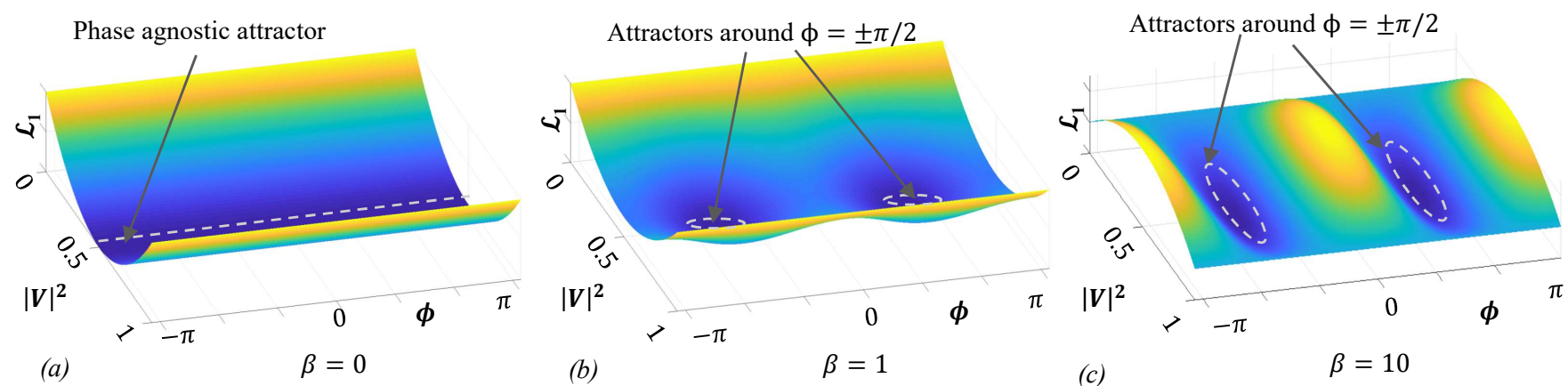

Figure 4. Cost function $\mathcal{L}_{1}$ plotted for different values of the hyperparameter $\beta$ : (a) $\beta=0$; (b) $\beta=1$ and (c) $\beta=10$.

Table 2: Complex growth transform dynamical system (Proof in Appendix C)

- For an optimization problem of the form:

$$
\begin{gathered}
\min _{\left\{\left|V_{i}\right|,\left|I_{i}\right|, \phi_{i}\right\}} \mathcal{L}\left(\left\{\left|V_{i}\right|,\left|I_{i}\right|, \phi_{i}\right\}\right)=\mathcal{H}\left(\left\{\left|V_{i}\right|,\left|I_{i}\right|\right\}\right)+\beta \sum_{i=1}^{N}\left|V_{i}\right|^{2}\left|I_{i}\right|^{2} \cos ^{2} \phi_{i} \\
\text { s.t. } \quad \sum_{i=1}^{N}\left(\left|V_{i}\right|^{2}+\left|I_{i}\right|^{2}\right)=1, \quad\left|\phi_{i}\right| \leq \pi \quad \forall i=1, \ldots, N, \beta \geq 0
\end{gathered}
$$

- If $\mathcal{H}\left(\left\{\left|V_{i}\right|,\left|I_{i}\right|\right\}\right)$ is Lipschitz continuous in the domain $D=\left\{\left|V_{i}\right|,\left|I_{i}\right|: \sum_{i=1}^{N}\left(\left|V_{i}\right|^{2}+\left|I_{i}\right|^{2}\right)=1\right\}$, the following system of nonlinear dynamical equations

$$
\begin{aligned}
\frac{\partial V_{i}(t)}{\partial t} & =j \omega \sigma_{V_{i}}(t) V_{i}(t)-\Delta \sigma_{V_{i}}(t) V_{i}(t), \\
\frac{\partial I_{i}(t)}{\partial t} & =j\left(\omega+\omega_{\phi_{i}}\right) \sigma_{I_{i}}(t) I_{i}(t)-\Delta \sigma_{I_{i}}(t) I_{i}(t), \\
\text { and } \tau_{i} \omega_{\phi_{i}}+\phi_{i}(t) & =g_{\phi_{i}}(t), \quad \forall i=1, \ldots, N \\
\text { ensures that } \frac{\partial \mathcal{L}}{\partial t} \leq 0 &
\end{aligned}
$$

where $\sigma_{V_{i}}(t)=\sqrt{\frac{1}{V_{i}^{*} \eta}\left(-\frac{\partial \mathcal{L}}{\partial V_{i}}+\lambda V_{i}^{*}\right)}, \sigma_{I_{i}}(t)=\sqrt{\frac{1}{I_{i}^{*} \eta}\left(-\frac{\partial \mathcal{L}}{\partial I_{i}}+\lambda I_{i}^{*}\right)}, \omega_{\phi_{i}}=\frac{d \phi_{i}(t)}{d t}, \Delta \sigma_{V_{i}}(t)=1-\sigma_{V_{i}}(t)$, $\Delta \sigma_{I_{i}}(t)=1-\sigma_{I_{i}}(t)$ and $g_{\phi_{i}}(t)=\pi \frac{\lambda \phi_{i}-\pi \frac{\partial \mathcal{L}}{\partial \phi_{i}}}{-\phi_{i} \frac{\partial \mathcal{L}}{\partial \phi_{i}}+\lambda \pi}$, with $\eta=\sum_{k=1}^{N}\left(V_{k}\left[-\frac{\partial \mathcal{L}}{\partial V_{k}}+\lambda V_{k}^{*}\right]+I_{k}\left[-\frac{\partial \mathcal{L}}{\partial I_{k}}+\lambda I_{k}^{*}\right]\right), \omega$ is an arbitrary angular frequency, and $\tau_{i}$ is the time-constant associated with the evolution of $\phi_{i}$.

\section{COMPlEX GROWTH TRANSFORMS}

The problem in Equation (10) involves complex phasors and hence entails the use of learning models operating in the complex domain for reaching the optimal solution. To this end, in this section, we propose a dynamical system that can be used to solve this optimization problem. The main result is summarized in Table 2 and the details of the proof is given in Appendix C.

Theorem 1: The system of nonlinear dynamical equations given by Equations (15)-(18) in Table 2 converge to the optimal point of Equation (14) in the steady state, with zero energy dissipation i.e., $\sum_{n=1}^{N}\left|V_{i}\right|\left|I_{i}\right| \cos \phi_{i}=0$ (Proof given in Appendix C) .
Consider an optimization framework which is an multivariable extension of Example 1, given by Equations (12)(13):

$$
\begin{aligned}
\min _{\left\{\left|V_{i}\right|,\left|I_{i}\right|, \phi_{i}\right\}} & \mathcal{L}_{N}=\sum_{i=1}^{N}\left(\left|V_{i}\right|^{2}-\left|I_{i}\right|^{2}\right)^{2}+\beta \sum_{i=1}^{N}\left|V_{i}\right|^{2}\left|I_{i}\right|^{2} \cos ^{2} \phi_{i} \\
\text { s.t. } & \sum_{i=1}^{N}\left(\left|V_{i}\right|^{2}+\left|I_{i}\right|^{2}\right)=1, \quad\left|\phi_{i}\right| \leq \pi \forall i
\end{aligned}
$$

The optimal solution is reached when $\phi_{i}= \pm \frac{\pi}{2} \forall i$, which implies $\mathcal{L}_{N}=0$. For the sake of comparison, we will consider two variants: (a) the non-resonant model $\left(\mathcal{M}_{n r}\right)$ where $\beta=0$, and (b) the resonant model $\left(\mathcal{M}_{r}\right)$, where $\beta \neq 0$. 
Figures 5(a) and (b) show a comparison of the cost $\mathcal{H}_{N}$ and the regularization metric $\mathcal{D}_{N}\left(\sum_{i=1}^{N}\left|V_{i}\right|^{2}\left|I_{i}\right|^{2}\right.$ for $\mathcal{M}_{n r}$ and $\sum_{i=1}^{N}\left|V_{i}\right|^{2}\left|I_{i}\right|^{2} \cos ^{2} \phi_{i}$ for $\mathcal{M}_{r}$ ), for $N=5$ and $\omega=\pi / 10$. In the case of $\mathcal{M}_{n r}, \mathcal{L}_{N}=\mathcal{H}_{N}$ and in the case of $\mathcal{M}_{r}$, $\mathcal{L}_{N}=\mathcal{H}_{N}+\beta \mathcal{D}_{N}$, with $\beta=1$. The solid line indicates the mean response across 10 trials with random initializations of $V_{i}, I_{i}$ and $\phi_{i}$. The shaded region shows the standard deviation across the trials in each case. Also shown in Figure 5(b) are the true active-power dissipation profiles $\left(\sum_{n=1}^{N}\left|V_{i}\right|\left|I_{i}\right|\right.$ for $\mathcal{M}_{n r}$ and $\sum_{n=1}^{N}\left|V_{i}\right|\left|I_{i}\right| \cos \phi_{i}$ for $\mathcal{M}_{r}$ ) over the 10 trials.

It can be observed that under steady-state conditions, the model $\mathcal{M}_{n r}$ dissipates power. However for the model $\mathcal{M}_{r}$ the steady-state active-power goes to zero. This is illustrated in Figure 5(b). Figure 5(c) shows the initial phasor configuration for the currents and voltages at each node of the network for a single trial for $\mathcal{M}_{r}$. Here, we assume the voltage phasor $V_{i}$ to be aligned with the horizontal axis, and the current phasor $I_{i}$ to be at an angle $\phi_{i}$ with respect to the horizontal, $\forall i$. Figure 5.d) shows the steady-state phasor configuration for $\mathcal{M}_{r}$ for the same trial. The voltage and current phasors are orthogonal to each other for all the nodes, thus verifying the zero activepower dissipation.

In the next set of experiments, we annealed the hyperparameter $\beta$ and evaluated its impact on the active-power dissipation metric $\mathcal{D}_{N}$ and the convergence of the object function $\mathcal{H}_{N}$ for the model $\mathcal{M}_{r}$. Figure 6 presents a comparison of the performance of $\mathcal{M}_{r}$ for different choices of annealing schedule for $\beta$, with the angular frequency $\omega=\pi / 10$ as before. Figure 6 a) shows the time evolution of the objective function $\mathcal{H}_{N}$, 6 (b) shows the time evolution of the dissipation metric $\mathcal{D}_{N}$ and 6.c) shows the annealing schedules adopted for $\beta$. In all the cases, the optimization process starts after time $t=0.1$ a.u. from the onset of the simulation. The curves corresponding to $\beta=1$ denote the case when $\beta$ takes a constant value from $t=0.1$ a.u.; $\beta=$ logistic corresponds to the case when $\beta$ is slowly increased from $\beta_{\min }=0$ following a logistic curve of the form $\beta(t)=\beta_{\min }+\frac{\beta_{\max }-\beta_{\min }}{1+\exp \left(-k\left(t+t_{0}\right)\right)}$ from $t=0.1$ a.u., and takes on a maximum value of $\beta_{\max }=1(k$ and $t_{0}$ are hyperparameters determining the steepness and midpoint of the sigmoid respectively); $\beta=$ switching corresponds to the case when $\beta$ switches from a minimum value $\left(\beta_{\min }=0\right)$ to a maximum value $\left(\beta_{\max }=1\right)$ at $t=0.3 a . u$., after the system has converged to the optimal solution. We can observe that in all of the cases, the model converges to the optimal solution, irrespective of the choice of $\beta$. However, different annealing schedules for $\beta$ lead to different activepower dissipation profiles. For example, a constant value of $\beta$ throughout the duration of the experiment would lead to faster minimization of the active-power dissipation metric, but at the cost of slower convergence. The opposite trend can be seen when $\beta$ is slowly annealed to a sufficiently high value throughout the course of the optimization. The annealing schedule thus acts as a trade-off between the speed of convergence, and the rate of minimization of the active power.

\section{A. Model Properties and Extensions}

The dynamical system represented by Equations (15)- 18 and the resonant optimization framework also exhibits the following properties and extensions:

1) Energy constraints can be imposed over subgroups of nodes in the network: We can have the reactive energy conservation constraint between subgroups of nodes, instead of on the network as a whole, i.e., $\sum_{i=1}^{N_{k}}\left(\left|V_{i k}\right|^{2}+\right.$ $\left.\left|I_{i k}\right|^{2}\right)=1 \forall k=1, \ldots, M$. Here $M=$ number of subgroups and $N_{k}=$ number of nodes in the $k^{\text {th }}$ subgroup. The update equations in this case are given by:

$$
\begin{gathered}
\frac{\partial V_{i k}(t)}{\partial t}=j \omega_{k} \sigma_{V_{i k}}(t) V_{i k}(t)-\Delta \sigma_{V_{i k}}(t) V_{i k}(t) \\
\frac{\partial I_{i k}(t)}{\partial t}=j\left(\omega_{k}+\omega_{\phi_{i k}}\right) \sigma_{I_{i k}}(t) I_{i k}(t)-\Delta \sigma_{I_{i k}}(t) I_{i k}(t) \\
\tau_{i k} \omega_{\phi_{i k}}+\phi_{i k}(t)=g_{\phi_{i k}}(t) \quad \forall i, k
\end{gathered}
$$

where $\omega_{k}$ is the constant angular frequency of the $k^{\text {th }}$ subgroup of nodes, and $\omega_{\phi_{i k}}=\frac{d \phi_{i k}(t)}{d t}$.

2) System dynamics and reactive-energy constraints remain invariant under the imposition of a global phase: The network dynamics remain invariant to the imposition of a global phase component on all the network variables, and the conservation constraint is also satisfied in this case. The governing equations are given by:

$$
\begin{aligned}
\frac{\partial V_{i}(t)}{\partial t} & =j\left(\omega+\omega_{\phi_{g}}\right) \sigma_{V_{i}}(t) V_{i}(t)-\Delta \sigma_{V_{i}}(t) V_{i}(t), \\
\frac{\partial I_{i}(t)}{\partial t} & =j\left(\omega+\omega_{\phi_{g}}+\omega_{\phi_{i}}\right) \sigma_{I_{i}}(t) I_{i}(t)-\Delta \sigma_{I_{i}}(t) I_{i}(t),
\end{aligned}
$$

where $\phi_{g}$ is the global phase and $\omega_{\phi_{g}}=\frac{d \phi_{g}}{d t}$.

3) Reactive-energy constraints remain invariant with varying network dynamics under the imposition of a relative phase: The conservation constraints are satisfied on the introduction of a relative phase component between the voltage and current phasors of each node, even though the overall network dynamics change. The governing equations are given by:

$$
\begin{aligned}
& \frac{\partial V_{i}(t)}{\partial t}=j\left(\omega+\omega_{\phi_{V_{i}}}\right) \sigma_{V_{i}}(t) V_{i}(t)-\Delta \sigma_{V_{i}}(t) V_{i}(t), \\
& \frac{\partial I_{i}(t)}{\partial t}=j\left(\omega+\omega_{\phi_{I_{i}}}+\omega_{\phi_{i}}\right) \sigma_{I_{i}}(t) I_{i}(t)-\Delta \sigma_{I_{i}}(t) I_{i}(t),
\end{aligned}
$$

where $\phi_{i}=\phi_{I_{i}}-\phi_{V_{i}}$ is the relative external phase shift applied between the voltage and current phasors of the $i^{\text {th }}$ node, $\omega_{\phi_{V_{i}}}=\frac{d \phi_{V_{i}}}{d t}$ and $\omega_{\phi_{I_{i}}}=\frac{d \phi_{I_{i}}}{d t}$. 


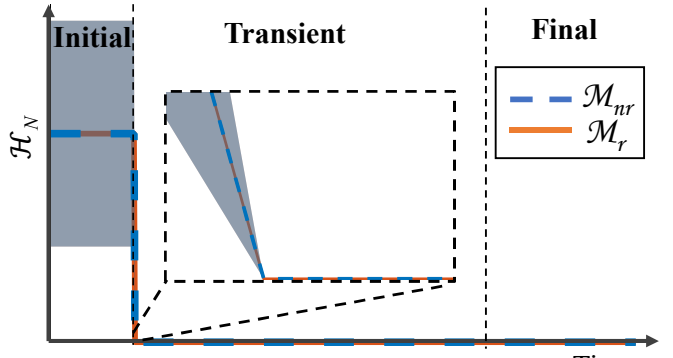

(a)

Time

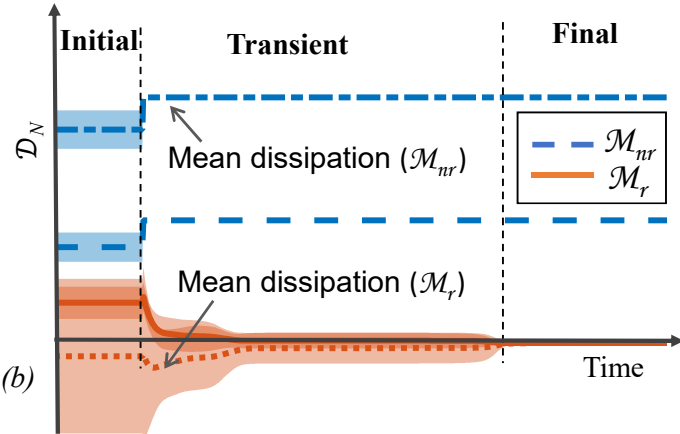

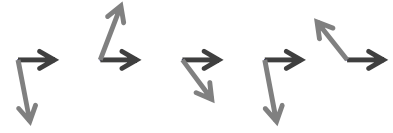

(c) Initial Phasor $\left(\mathcal{M}_{r}\right)$

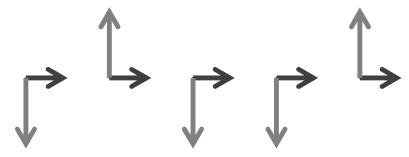

(d) Final Phasor $\left(\mathcal{M}_{r}\right)$

Figure 5. Comparison of the resonant optimization model $\left(\mathcal{M}_{r}\right)$ with its non-resonant variant $\left(\mathcal{M}_{n r}\right)$ for a quadratic objective function $\mathcal{L}_{N}$ shown in Equation 19 . For this experiment, $N=5$, input frequency $\omega=\pi / 10$, and the mean response is estimated over 10 trials with random initializations of $V_{i}, I_{i}, \phi_{i} \forall i=1, \ldots, 5$ : (a) comparison of the time-evolution of $\mathcal{H}_{N}$ for $\mathcal{M}_{n r}$ and $\mathcal{M}_{r}$, (b) comparison of the time-evolution of $\mathcal{D}_{N}\left(\sum_{i=1}^{N}\left|V_{i}\right|^{2}\left|I_{i}\right|^{2}\right.$ for $\mathcal{M}_{n r}$ and $\sum_{i=1}^{N}\left|V_{i}\right|^{2}\left|I_{i}\right|^{2} \cos ^{2} \phi_{i}$ for $\mathcal{M}_{r}$ ). For all the curves, the line indicates the mean response, while the shaded region shows the standard deviation about the mean across the 10 trials. The true dissipation $\left(\sum_{n=1}^{N}\left|V_{i}\right|\left|I_{i}\right|\right.$ for $\mathcal{M}_{n r}$ and $\sum_{n=1}^{N}\left|V_{i}\right|\left|I_{i}\right| \cos \phi_{i}$ for $\left.\mathcal{M}_{r}\right)$ over the 10 trials are also shown in the figure; Phasor representations of the LC tank voltages and currents for a single trial : (c) initial configuration and (d) final configuration for $\mathcal{M}_{r}$. For $\mathcal{M}_{r}$, $\beta=1$ for all the trials.

4) The model is dissipative and converges to limit cycle oscillations in steady state: The second order time derivatives of Equations (15) and (16) lead to the following:

$$
\begin{aligned}
& \frac{\partial^{2} V_{i}}{\partial t^{2}}=j \omega \sigma_{V_{i}} \dot{V}_{i}+j \omega V_{i} \dot{\sigma}_{V_{i}}-\Delta \sigma_{V_{i}} \dot{V}_{i}+\sigma_{V_{i}} V_{i} \\
& =\underbrace{-\omega^{2} \sigma_{V_{i}}^{2} V_{i}}_{\text {limit cycles }}+ \\
& \underbrace{[1+j \omega] \dot{\sigma}_{V_{i}} V_{i}-2 j \omega \sigma_{V_{i}} \Delta \sigma_{V_{i}} V_{i}+\left(\Delta \sigma_{V_{i}}\right)^{2} V_{i}}_{\text {dissipation }}, \\
& \frac{\partial^{2} I_{i}}{\partial t^{2}}=j \omega_{\phi_{i}}^{\prime} \sigma_{I_{i}} \dot{I}_{i}+j \omega_{\phi_{i}}^{\prime} I_{i}{\dot{\sigma_{i}}}_{I_{i}}-\Delta \sigma_{I_{i}} \dot{I}_{i}+\sigma_{I_{i}} I_{i} \\
& =\underbrace{-\left(\omega_{\phi_{i}}^{\prime}\right)^{2} \sigma_{I_{i}}^{2} I_{i}}_{\text {limit cycles }}+ \\
& \underbrace{\left[1+j \omega_{\phi_{i}}^{\prime}\right] \dot{\sigma}_{I_{i}} I_{i}-2 j \omega_{\phi_{i}}^{\prime} \sigma_{I_{i}} \Delta \sigma_{I_{i}} I_{i}+\left(\Delta \sigma_{I_{i}}\right)^{2} I_{i}}_{\text {dissipation }},
\end{aligned}
$$

where $\omega_{\phi_{i}}^{\prime}=\omega+\omega_{\phi_{i}}$. The first terms in the RHS of Equations (24) and (25) correspond to stable limit cycle oscillations of all the phasors, while the other terms correspond to the dissipative effects in the network. This demonstrates that the network as a whole is essentially a coupled dissipative system that is capable of selfsustained oscillations under steady-state. Each individual state variable describing the network thus returns to the same position in its respective limit cycle at regular intervals of time, even when subjected to small perturbations.

\section{Resonant Machine Learning Framework}

In this section, we show how the framework introduced in Section III can be applied for constructing resonant machine learning networks. In general, the framework can be applied to any learning network that optimizes a cost function defined over a set of learning variables $\alpha_{i}$ as

$$
\begin{gathered}
\min _{\left\{\alpha_{i}\right\}} \mathcal{H}\left(\left\{\alpha_{i}\right\}\right)+h \Psi\left(\left\{\alpha_{i}\right\}\right) \\
\text { s.t. } \quad \sum_{i=1}^{N} \alpha_{i}=1, \quad \alpha_{i} \geq 0 \quad \forall i=1, \ldots, N .
\end{gathered}
$$

Here $\mathcal{H}\left(\left\{\alpha_{i}\right\}\right)$ represents a loss-function [28] which depends on the learning problem (e.g. supervised, unsupervised or semi-supervised) and the dataset under consideration (e.g., training data). The second term $\Psi(\cdot)$ in the objective function is any linear or nonlinear function which represents either (a) a regularization function, or (b) a penalty function used to satisfy optimization constraints. $h$ is a hyperparameter which acts as a trade-off between $\mathcal{H}(\cdot)$ and $\Psi(\cdot)$. Because $\alpha_{i}$ could be viewed as probability measure, the optimization framework in Equation 26) naturally lends itself to probabilistic learning models [29]-[31].

The above problem can be mapped to the resonant learning framework in Section III by substituting $\alpha_{i}=\left|V_{i}\right|^{2}+\left|I_{i}\right|^{2}$, to arrive at the following problem:

$$
\begin{array}{cl}
\min _{\left\{\left|V_{i}\right|,\left|I_{i}\right|, \phi_{i}\right\}} \quad \mathcal{H}\left(\left\{\left|V_{i}\right|,\left|I_{i}\right|\right\}\right)+h \Psi\left(\left\{\left|V_{i}\right|,\left|I_{i}\right|\right\}\right) \\
+\beta \sum_{i=1}^{N}\left|V_{i}\right|^{2}\left|I_{i}\right|^{2} \cos ^{2} \phi_{i} \\
\text { s.t. } \quad \sum_{i=1}^{N}\left(\left|V_{i}\right|^{2}+\left|I_{i}\right|^{2}\right)=1, \quad|\phi| \leq \pi \quad \forall i=1, \ldots, N
\end{array}
$$

Note that non-probabilistic learning problems can also be mapped to the probabilistic framework by imposing an additional constraint, as discussed in Appendix A. 

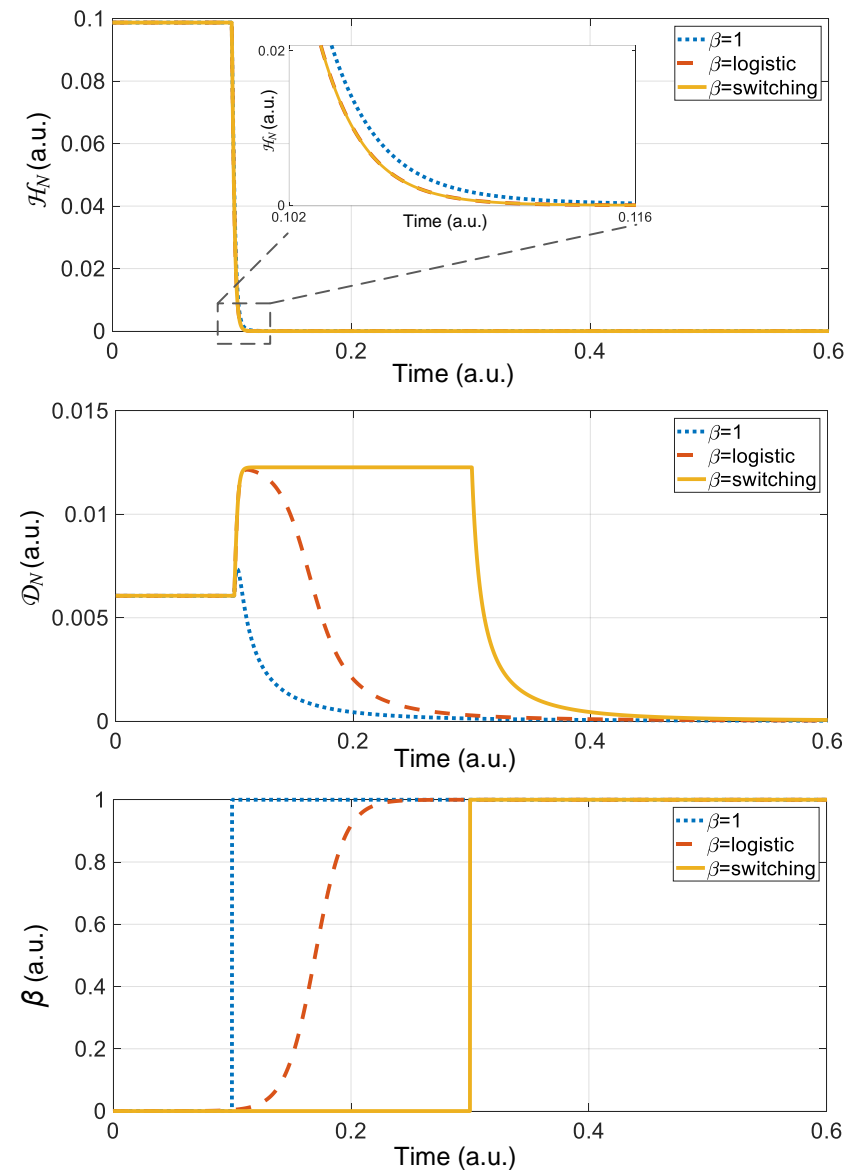

Figure 6. Comparison of the performance of the resonant model $\mathcal{M}_{r}$ for different choices of annealing schedule for $\beta(N=5, \omega=\pi / 10)$ : (a) time evolution of $\mathcal{H}_{N}$ (inset shows a zoomed in view of the cost evolution in the transient phase), (b) time evolution of $\mathcal{D}_{N}$ and (c) time evolution of $\beta$. In al the cases, the optimization process starts after 0.1 a.u. from the onset of the simulation. The curves corresponding to $\beta=1$ denotes the case when $\beta$ takes a constant value from $t=0.1$ a.u.; $\beta=$ logistic corresponds to the cases when $\beta$ is slowly increased following a logistic curve from $t=0.1$ a.u., and takes on a maximum value of $\beta=1 ; \beta=$ switching corresponds to the case when $\beta$ switches from a minimum value $(=0)$ to a maximum value $(=1)$ at $t=0.3$ a.u., after the system has converged to the optimal solution.

\section{A. One-class resonant SVM}

We now show how the framework in Equation (27) can be used to design a resonant one-class SVM.

The solution of a generic one-class SVM is obtained by solving the following optimization problem [8], [32], [33]:

$$
\begin{gathered}
\min _{\left\{\alpha_{i}\right\}} \frac{1}{2} \sum_{i=1}^{N} \sum_{j=1}^{N} \alpha_{i} K\left(\mathbf{x}_{i}, \mathbf{x}_{j}\right) \alpha_{j} \\
\text { s.t. } \quad \sum_{i=1}^{N} \alpha_{i}=1, \quad 0<\alpha_{i}<\frac{1}{\nu N} \quad \forall i=1, \ldots, N
\end{gathered}
$$

where $\mathbf{X}=\left[\mathbf{x}_{1}, \ldots, \mathbf{x}_{\mathbf{i}}, \ldots, \mathbf{x}_{\mathbf{N}}\right] \in \mathbb{R}^{N \times D}$ is the $D$-dimensional input dataset of size $N, \nu \in\{0,1\}$ is a parameter which controls the size of the decision surface, $K(\cdot, \cdot)$ is a positive definite Kernel function satisfying Mercer's conditions, and $\alpha_{i}$ 's are the learning parameters. The optimization problem above can be reformulated by replacing the inequality constraint with a smooth penalty or loss function $\Psi(\cdot)$ like the logarithmic barrier, e.g., $\Psi\left(\alpha_{i}, \nu\right)=-\log \left(\frac{1}{\nu N}-\alpha_{i}\right)$.

$$
\begin{gathered}
\min _{\left\{\alpha_{i}\right\}} \mathcal{H}=\frac{1}{2} \sum_{i=1}^{N} \sum_{j=1}^{N} \alpha_{i} K\left(\mathbf{x}_{i}, \mathbf{x}_{j}\right) \alpha_{j}+h \sum_{i=1}^{N} \Psi\left(\alpha_{i}, \nu\right) \\
\text { s.t. } \quad \sum_{i=1}^{N} \alpha_{i}=1,
\end{gathered}
$$

The parameter $h$ determines the steepness of the penalty function, where a lower value of $h$ implies an almost-accurate inequality constraint.

An equivalent complex-domain representation in terms of voltages and currents in an LC network can be arrived at if we consider $\alpha_{i}=\left|V_{i}\right|^{2}+\left|I_{i}\right|^{2} \forall i$. In this case, we consider that the network is globally energy constrained, and all the individual units in the network have the same frequency $\omega$. The redefined learning problem is as follows:

$$
\begin{gathered}
\min _{\left\{\left|V_{i}\right|,\left|I_{i}\right|\right\}} \mathcal{H}=\frac{1}{2} \sum_{i=1}^{N} \sum_{j=1}^{N}\left(\left|V_{i}\right|^{2}+\left|I_{i}\right|^{2}\right) K\left(\mathbf{x}_{i}, \mathbf{x}_{j}\right) \\
\times\left(\left|V_{j}\right|^{2}+\left|I_{j}\right|^{2}\right)+h \sum_{i=1}^{N} \Psi\left(\left|V_{i}\right|,\left|I_{i}\right|, \nu\right) \\
\text { s.t. } \quad \sum_{i=1}^{N}\left(\left|V_{i}\right|^{2}+\left|I_{i}\right|^{2}\right)=1 .
\end{gathered}
$$

Introducing the active-power dissipation regularization we arrive at the following problem:

$$
\begin{gathered}
\min _{\left\{\left|V_{i}\right|,\left|I_{i}\right|, \phi_{i}\right\}} \mathcal{L}=\frac{1}{2} \sum_{i=1}^{N} \sum_{j=1}^{N}\left(\left|V_{i}\right|^{2}+\left|I_{i}\right|^{2}\right) K\left(\mathbf{x}_{i}, \mathbf{x}_{j}\right) \\
\times\left(\left|V_{j}\right|^{2}+\left|I_{j}\right|^{2}\right)+h \sum_{i=1}^{N} \Psi\left(\left|V_{i}\right|,\left|I_{i}\right|, \nu\right) \\
+\beta \sum_{i=1}^{N}\left|V_{i}\right|^{2}\left|I_{i}\right|^{2} \cos ^{2} \phi_{i} \\
\text { s.t. } \quad \sum_{i=1}^{N}\left(\left|V_{i}\right|^{2}+\left|I_{i}\right|^{2}\right)=1, \quad\left|\phi_{i}\right| \leq \pi \forall i .
\end{gathered}
$$

The update equations in this case are of the form shown in Equations (15)-(18). Figure 7] shows a comparison of the active and reactive power metrics of each node of the non-resonant model $\mathcal{M}_{n r}$ and the resonant model $\mathcal{M}_{r}$ for a synthetic one-class SVM problem on a two-dimensional dataset (Dataset I). The dataset was generated by uniformly selecting 300 random points within a circle having a fixed radius. Here $N=300, \nu=0.1, \omega=\pi / 4$, with random initializations for $V_{i}, I_{i}, \phi_{i} \forall i=1, \ldots, N$. A constant value of the regularization hyperparameter $\beta=1$ was considered throughout the duration of the optimization process for $\mathcal{M}_{r}$. Figures 7(a) and (c) show the values of the active power metric $\left(=\left|V_{i}\right|\left|I_{i}\right|\right)$ at each node in the initial and final stages respectively for $\mathcal{M}_{n r}$, while Figures 7 (b) and (d) show the values of the reactive power metric $(=0)$ at each node in the initial and final stages respectively for $\mathcal{M}_{n r}$. Similarly, 


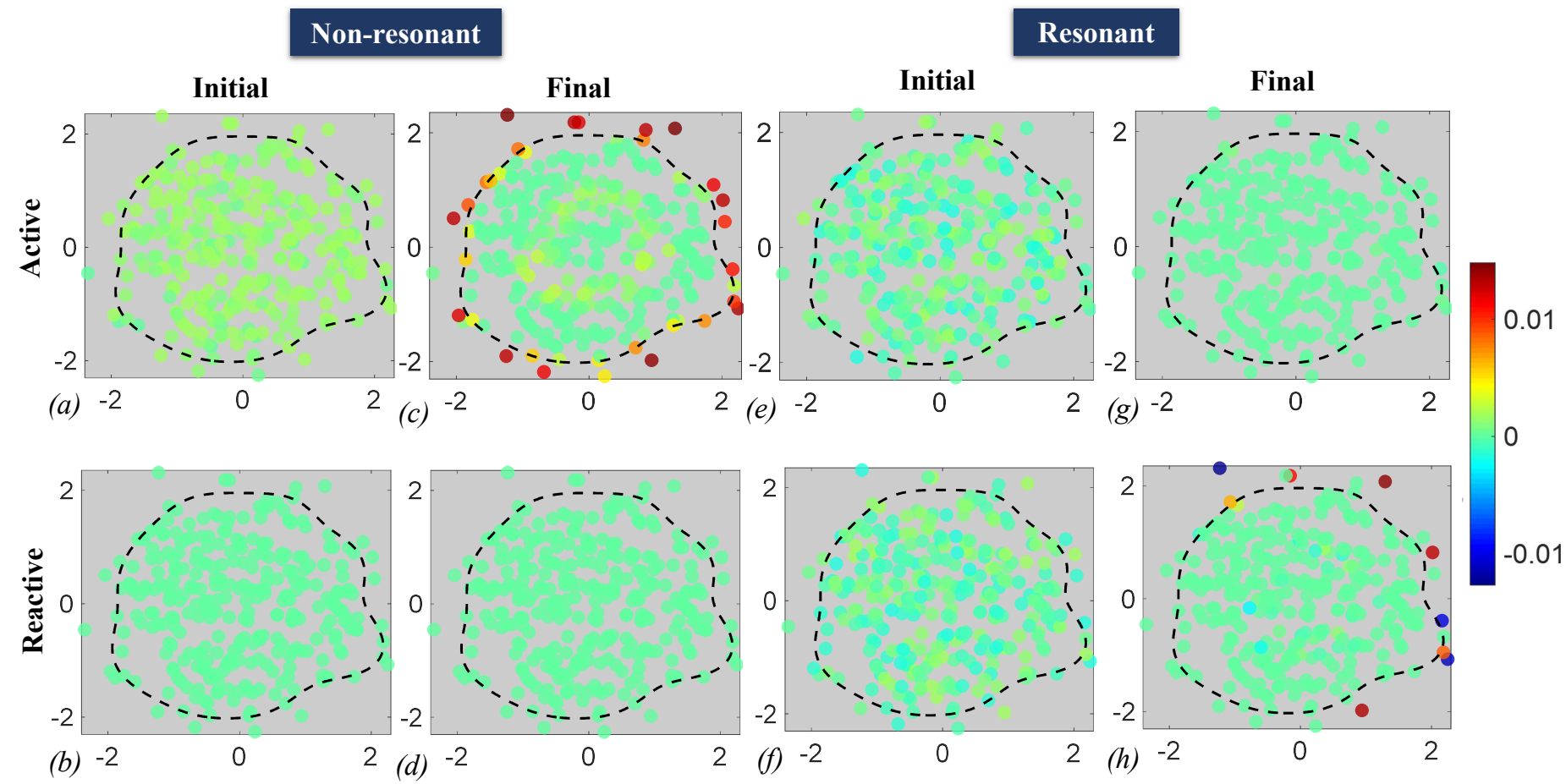

Figure 7. Comparison of the active and reactive power dissipated at each node for the non-resonant model $\mathcal{M}_{n r}$ and the resonant model $\mathcal{M}_{r}$ for the synthetic one-class SVM problem on a two-dimensional dataset (Dataset I), with $N=300, \nu=0.1, \omega=\pi / 4$, and random initializations for $V_{i}, I_{i}, \phi_{i} \forall i=1, \ldots, N$ : (a), (c): the values of the active power metric $\left(=\left|V_{i}\right|\left|I_{i}\right|\right)$ at each node in the initial and final stages respectively for $\mathcal{M}_{n r}$; (b), (d): the values of the reactive power metric $(=0)$ at each node in the initial and final stages respectively for $\mathcal{M}_{n r} ;(\mathrm{e}),(\mathrm{g})$ : the values of the active power metric $\left(=\left|V_{i}\right|\left|I_{i}\right| \cos \phi_{i}\right)$ at each node in the initial and final stages respectively for $\mathcal{M}_{r}$; (f), (h): the values of the reactive power metric (=|V $V_{i}|| I_{i} \mid$ sin $\left.\phi_{i}\right)$ at each node in the initial and final stages respectively for $\mathcal{M}_{r}$. For both models, $K(\cdot, \cdot)$ was chosen to be a Gaussian kernel with kernel parameter $\sigma=1$, and $\beta=1$ throughout the optimization process for $\mathcal{M}_{r}$.

Figures 7 (e) and (g) show the values of the active power metric $\left(=\left|V_{i}\right|\left|I_{i}\right| \cos \phi_{i}\right)$ at each node in the initial and final stages respectively for $\mathcal{M}_{r}$ while Figures $7(\mathrm{f})$ and (h), finally, show the values of the reactive power metric $\left(=\left|V_{i}\right|\left|I_{i}\right| \sin \phi_{i}\right)$ at each node in the initial and final stages respectively for $\mathcal{M}_{r}$.

Figure 8 shows a comparison of the performance of the resonant model $\mathcal{M}_{r}$ for different choices of annealing schedules for $\beta$ on three different two-dimensional synthetic datasets for a simulation duration of $t=4$ a.u. In all cases, $N=$ $300, \nu=0.1, \omega=\pi / 20$, and $K(\cdot, \cdot)$ was chosen to be a Gaussian kernel with parameter values $\sigma=1,10$ and 20 respectively for synthetic Dataset I, II and III. Additionally for each dataset, $V_{i}, I_{i}, \phi_{i}$ were randomly initialized $\forall i=$ $1, \ldots, N$. Figure 8 (a) shows the contour plot with the decision boundary around the datapoints, along with the support vectors (SVs) for Dataset I, while Figures 8,b), (c) and (d) show the time evolutions of the cost $\mathcal{H}$, the dissipation metric $\mathcal{D}$ and the hyperparameter $\beta$ for different annealing schedules. The curves corresponding to $\beta=1$ and 10 denote the cases when $\beta$ takes a constant value throughout the simulation duration. $\beta=\operatorname{logistic}_{1}$ and $\beta=\operatorname{logistic}_{2}$ correspond to the cases when $\beta$ is slowly increased following a logistic curve of the form $\beta(t)=\beta_{\min }+\frac{\beta_{\max }-\beta_{\min }}{1+\exp \left(-k\left(t+t_{0}\right)\right)}$, and takes on maximum values of $\beta_{\max }=1$ and $\beta_{\max }=10$ respectively starting from $\beta_{\min }=0$. Finally, $\beta=$ switching corresponds to the case when $\beta$ switches from a minimum value $\left(\beta_{\min }=0\right)$ to a maximum value $\left(\beta_{\max }=10\right)$ at $t=2$ a.u., after the system has converged to the optimal solution. Figures 8(e)-(h) show similar plots on Dataset II, while Figures 8(i)-(l) show the plots corresponding to Dataset III. Dataset I, as described before, was generated by selecting 300 points uniformly within a two-dimensional circle having a fixed radius. Datasets II and III also consist of 300 data points generated using a Gaussian mixture model consisting of four different clusters, with a fixed cluster mean and variance associated with each cluster. Dataset II and III differ only in terms of the cluster means associated with their respective constituent clusters, while the cluster variances are the same for all the clusters for both the datasets. It can be seen that since the optimization problem is convex, the model always converges to the optimal solution for every dataset, irrespective of the the annealing schedule for $\beta$ or the dataset complexity. However, the dissipation profiles corresponding to a particular annealing schedule strongly depend on the complexity of the dataset. In general, however, higher values of $\beta$ would lead to lower dissipation profiles during the learning process. Also, the model shows a much slower convergence in terms of the actual objective function for a constant non-zero value of $\beta$ throughout the optimization compared to the case when $\beta$ is slowly annealed to a sufficiently high value. The choice of a proper annealing schedule for $\beta$ would thus involve a tradeoff between the speed of convergence and the rate of power dissipation. 


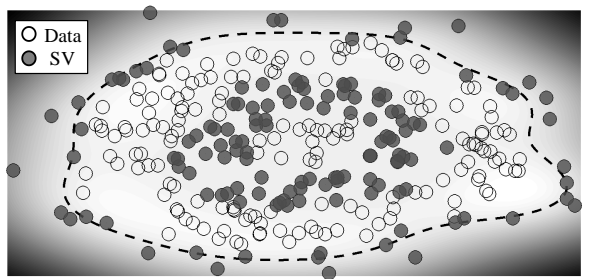

(a)
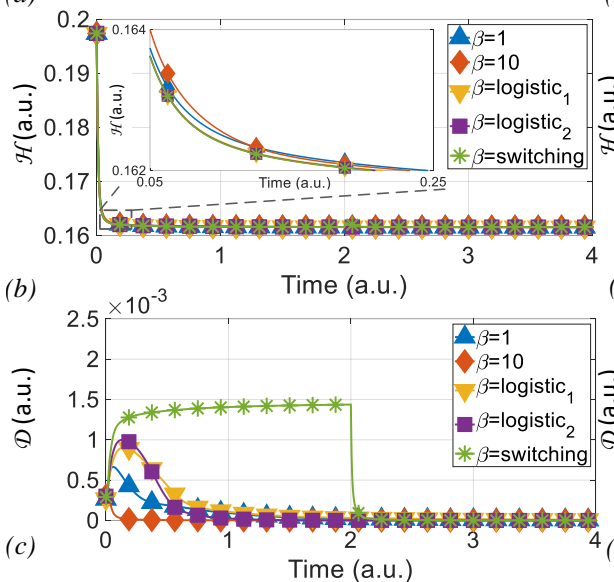

$(c)$
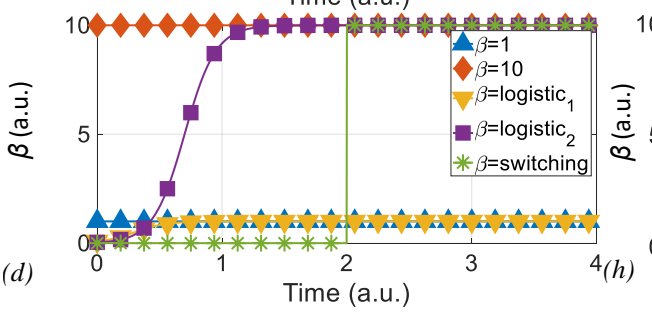

(e)
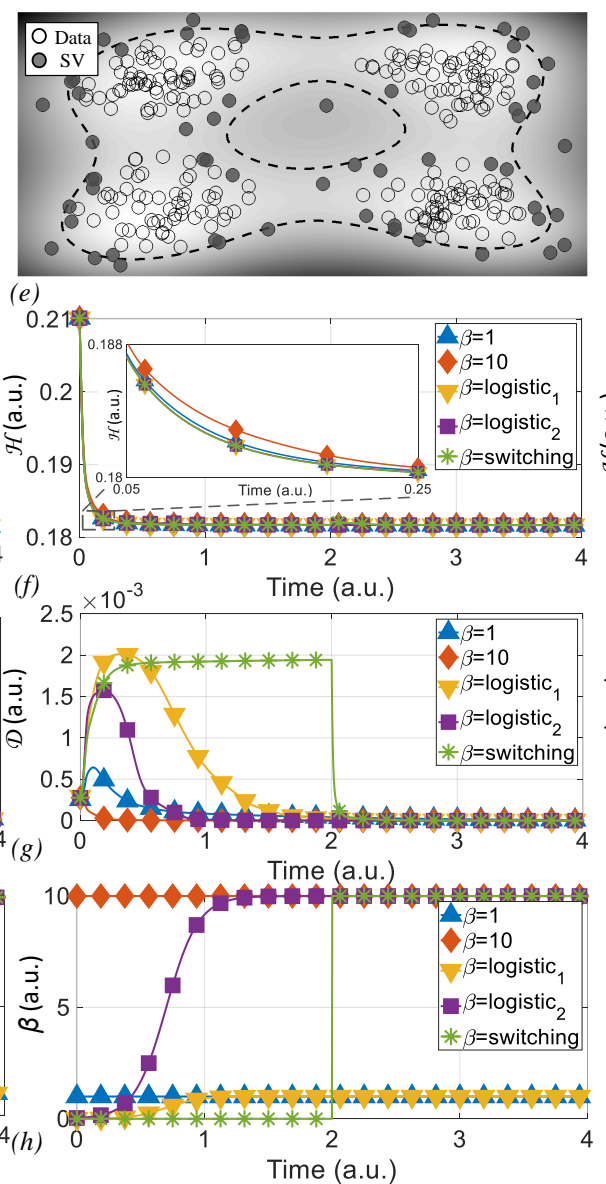

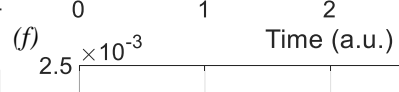

3

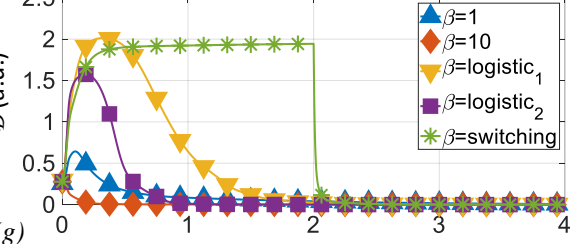

Time (a.u.) 3 , 4

(k)

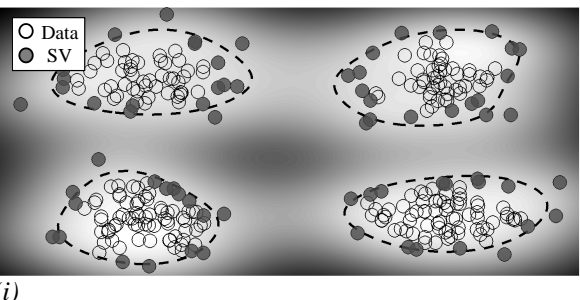

(i)

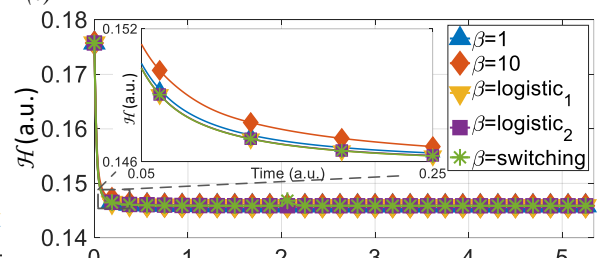$$
\text { (j) }
$$

(j) $25 \times 10^{-3} \quad$ Time (a.u.)
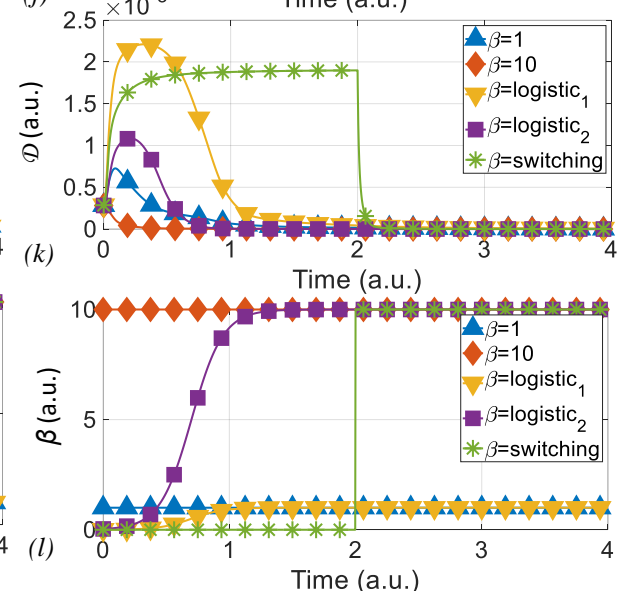

Figure 8. Comparison of the performance of the resonant model $\mathcal{M}_{r}$ for different choices of annealing schedules for $\beta$ for the one-class SVM problem, on three different two-dimensional synthetic datasets for a simulation duration of $t=4 a . u .$. In all cases, $N=300, \nu=0.1, \omega=\pi / 20$, and $K(\cdot, \cdot)$ was chosen to be a Gaussian kernel with parameter values $\sigma=1,10$ and 20 for synthetic Dataset I, II and III respectively. Additionally for each dataset, $V_{i}, I_{i}, \phi_{i}$ were randomly initialized $\forall i=1, \ldots, N$ : (a) contour plot with the decision boundary around the datapoints and support vectors (SVs); (b) time evolution of $\mathcal{H}$ (inset shows a zoomed in view of the cost evolution in the transient phase); (c) time evolution of $\mathcal{D}$ and (d) time evolution of $\beta$ for different annealing schedules. The curves corresponding to $\beta=1$ and 10 denote the cases when $\beta$ takes a constant value throughout the simulation duration; $\beta=$ logistic ${ }_{1}$ and $\beta=$ logistic $_{2}$ correspond to the cases when $\beta$ is slowly increased following a logistic curve, and takes on maximum values of $\beta_{\text {max }}=1$ and $\beta_{\text {max }}=10$ respectively; $\beta=$ switching corresponds to the case when $\beta$ switches from a minimum value $\left(\beta_{\min }=0\right)$ to a maximum value $\left(\beta_{\text {max }}=10\right)$ at $t=2$ a.u., after the system has converged to the optimal solution. (e)-(h) show similar plots on Dataset II, while (i)-(l) show the plots corresponding to Dataset III.

Figures 9 a) and (b) finally, show the robustness of the proposed framework to different initial conditions by providing a comparison of the time evolutions of the cost $\mathcal{H}$ and the dissipation metric $\mathcal{D}\left(\sum_{n=1}^{N}\left|V_{i}\right|^{2}\left|I_{i}\right|^{2}\right.$ for the non-resonant model $\mathcal{M}_{n r}$ and $\sum_{n=1}^{N}\left|V_{i}\right|^{2}\left|I_{i}\right|^{2} \cos ^{2} \phi_{i}$ for the resonant model $\mathcal{M}_{r}$ respectively), when applied to Dataset I. We show the results for 10 random initializations of $V_{i}, I_{i}$ and $\phi_{i}, \forall i$. In all cases, $\nu=0.1, \omega=\pi / 8$, and $K(\cdot, \cdot)$ was chosen to be a Gaussian kernel with parameter value $\sigma=1$. Note that even though the 10 trails had different initializations of $V_{i}, I_{i}$, they were chosen to have the same initial value of $\alpha_{i}^{\prime} \mathrm{s}$ in all cases, because of which there is no deviation between the cost evolution curves for both $\mathcal{M}_{n r}$ and $\mathcal{M}_{r}$. The dissipation evolution is however different across different trials for both the models. However, the dissipation attains a final value of zero for $\mathcal{M}_{r}$ for all the trials, while there is a finite dissipation for $\mathcal{M}_{n r}$ in all cases.

We also conducted experiments on real-life benchmark
Table 2: Performance on real benchmark datasets for $\nu=0.1$

\begin{tabular}{|c|c|c|c|c|}
\hline Dataset & Majority Class Size & Correct & Outliers & SVs \\
\hline Iris & $(50,4)$ & 48 & 2 & 8 \\
\hline Heart & $(139,13)$ & 1210 & 18 & 56 \\
\hline Diabetes & $(500,4)$ & 466 & 34 & 90 \\
\hline Ecoli & $(327,7)$ & 309 & 18 & 42 \\
\hline Adulta3a & $(2412,123)$ & 2111 & 301 & 356 \\
\hline Mammography & $(10923,6)$ & 9608 & 1315 & 5969 \\
\hline
\end{tabular}

datasets of varying sizes and dimensionality, considering the majority class as inliers in all cases. In all the experiments, we used similar parameter settings and annealing schedules as used in the experiments shown in Figure 8, and $\nu=0.1$. Table 2 shows the dataset description along with the performance (number of inliers, number of outliers and number of support 

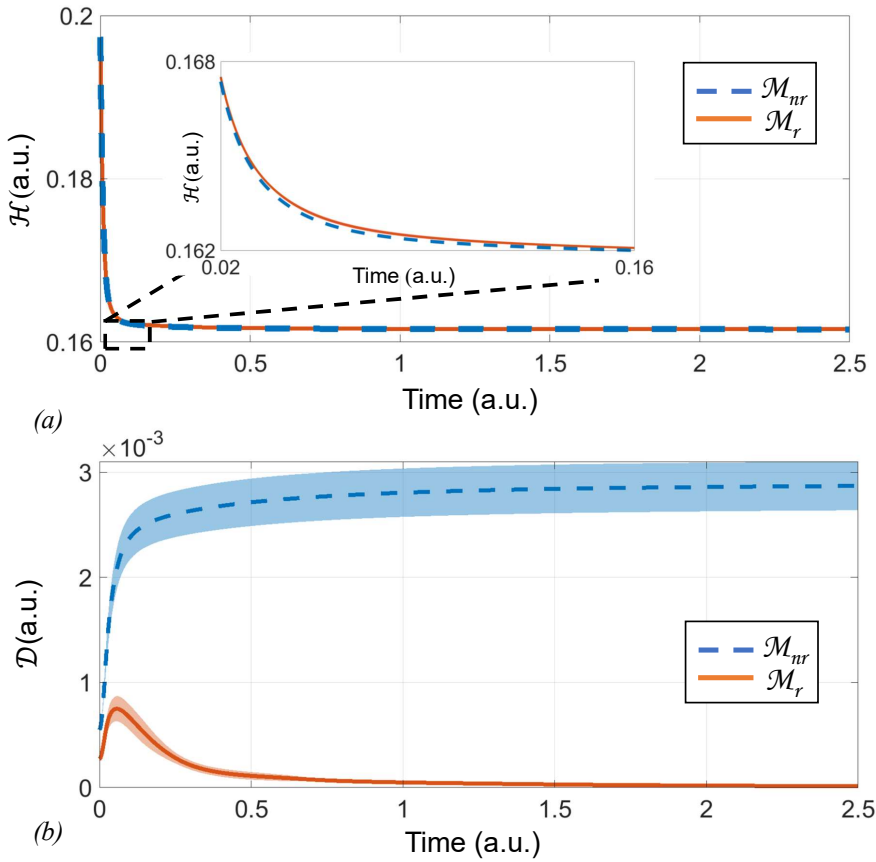

Figure 9. Robustness to random initialization: Comparison of (a) the timeevolution of the cost $\mathcal{H}$ and (b) the dissipation metric profile $\mathcal{D}\left(\sum_{n=1}^{N}\left|V_{i}\right|^{2}\left|I_{i}\right|^{2}\right.$ for the non-resonant model $\mathcal{M}_{n r}$ and $\sum_{n=1}^{N}\left|V_{i}\right|^{2}\left|I_{i}\right|^{2} \cos ^{2} \phi_{i}$ for the resonant model $\mathcal{M}_{r}$ respectively) for the synthetic one-class SVM problem on Dataset I for $N=300, \nu=0.1, \omega=\pi / 8$ over 10 random initializations for $V_{i}, I_{i}, \phi_{i} \forall i=1, \ldots, N$. The regularization parameter was chosen to be $\beta=1$ for the entire simulation duration of $t=4 a . u$. of the optimization process for $\mathcal{M}_{r}$. For all the curves, the solid line indicates the mean response, while the shaded region shows the standard deviation about the mean across the trials.

vectors) of the one-class SVM classifier for the following datasets: 'Iris' (inlier: class 'setosa'), 'Heart' (inlier: 'healthy' heart), 'Diabetes' (inlier: non-diabetic patients), 'Ecoli' (inlier: classes 'cp', 'im', 'pp', 'imU' and 'om'), 'Adulta3a' (inlier: individuals with income $\leq \$ 50 K$ ) and 'Mammography' (inlier: non-calcifications) [34], [35]. Figures 10] (a)-(f) show the dissipation profiles corresponding to different annealing schedules for the different datasets. It can be seen that an optimal choice of the annealing schedule depends on both the dataset size and complexity, even though the dissipation decreases over time for all the cases, irrespective of the schedule chosen.

Interestingly, the solution of the one-class SVM can be interpreted in terms of an electrical network as follows, as shown in Figure 11. (a) the support vectors have voltage and current magnitudes with a $\pm \pi / 2$ phase shift between them, hence can be interpreted as resonant LC tanks; and (b) the interior points well inside the boundary have both zero voltage and current magnitudes, and can be essentially treated as floating sinks.

\section{DisCUSSIONS AND CONCLUSIONS}

In this paper we proposed a complex-domain formulation of a machine learning network that ensures that the network's active power is dissipated only during the process of learning whereas the network's reactive power is maintained to be zero at all times. We showed that the active power dissipation during learning can be controlled using a phase regularization parameter. Also, the framework is robust to variations in the initial conditions and to the choice of the input/driving frequency $\omega$. The proposed approach thus provides a physical interpretation of machine learning algorithms, where the energy required for storing learned parameters is sustained by electrical resonances due to nodal inductances and nodal capacitances. Using the one-class support vector machine problem as a case study, we have shown how the steady state solution of a learning problem can be interpreted in terms of these nodal circuit elements.

Future directions involve exploring the implications of incorporating network dynamics in the free frequency variable $\omega$, and utilizing the phase information associated with each node in the learning process. Also, the experimental results presented in this paper were based on an unsupervised learning setting. Exploring an energy-efficient framework for supervised learning problems would also be a part of our future work.

In this paper we also proposed a dynamical system model based on complex-domain growth transforms. The formulation is general enough to be applicable to other complex-domain learning models [19]-[23]. Our proposed framework also preserves both the magnitude and phase information, and provides additional flexibilty compared to other complex domain learning models in terms of phase manipulation/cancellation [20].

In addition to implementing classic machine learning algorithms, the complex growth transform dynamical system can also be used for designing synchronized networks of coupled oscillators. Such networks can be potentially used for solving different computing tasks like optimization, pattern matching etc. as is achievable using coupled oscillatory computing models [36], [37]. An oscillator network designed in this fashion is capable of demonstrating stable, self-sustaining oscillatory dynamics, whereby the network can return to its initial stable limit cycle configuration following small perturbations, while simultaneously minimizing some underlying system-level objective function. The framework could also be used to study connections between learning and synchronization, or the emergence of a rhythmic periodic pattern exhibited by a group of coupled oscillators, which provides the key to understanding periodic processes pervading complex networks of different biological, physical, social and quantum ensembles [9], [38]. In this regard, the existing mathematical models for such collective behavior are mostly phenomenological or bottomup, and in general do not provide a network-level perspective of the underlying physical process. The proposed growthtransform formulation, thus, could provide new networklevel insights into the emergence of phase synchronization, phase cohesiveness and frequency synchronization in coupledoscillator networks.

Note here that since we are implicitly assuming an analog implementation, the learning network should converge to the steady-state solution of the optimization, where the time of convergence depends on the networks time-constant [39]. Hence the notion of time complexity is not well-defined. 

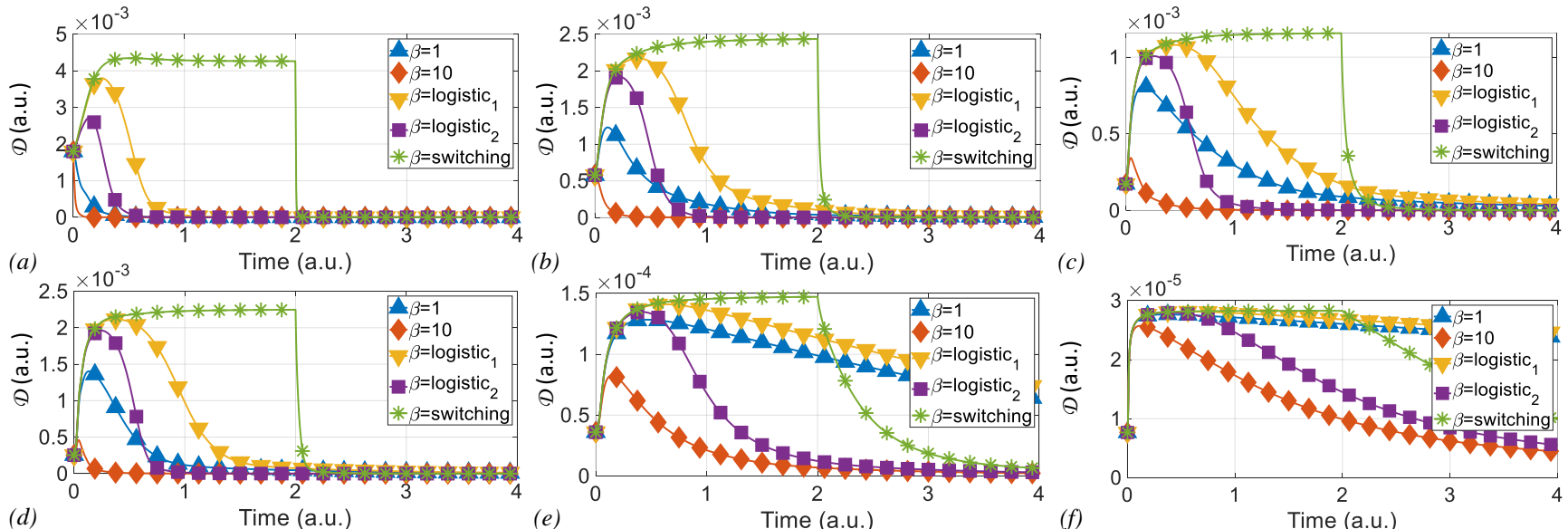

(c)

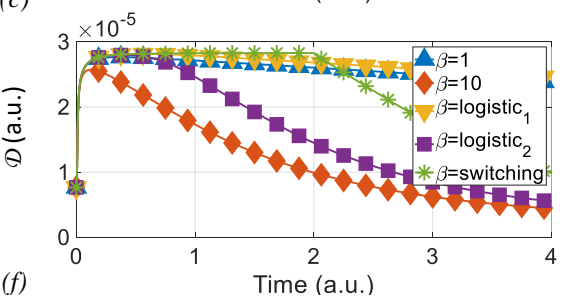

Figure 10. Comparison of the dissipation profiles of the resonant model $\mathcal{M}_{r}$ for different choices of annealing schedules for $\beta$ for different real-life benchmark datasets :(a) 'Iris'; (b) 'Heart'; (c) 'Diabetes; (d) 'Ecoli'; (e) 'Adulta3a'; and (f) 'Mammography'.

\section{Equivalent circuit representation}

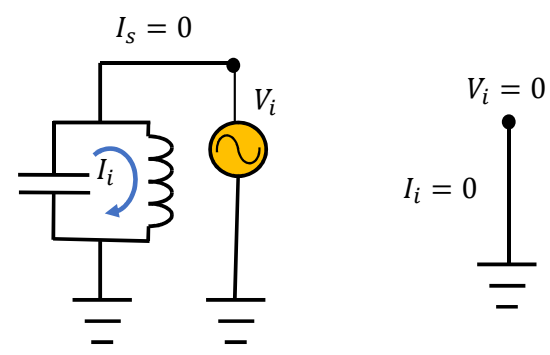

Phasor representation

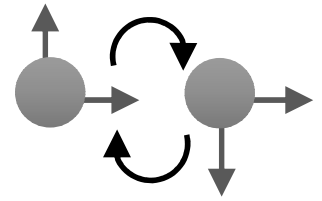

(a) Support Vectors (b) Interior Points

Figure 11. Circuit based and phasor based representations for a one-class SVM problem: (a) support vectors, corresponding to resonant LC tanks and (b) interior points, corresponding to sinks/ground $\left(V_{i}, I_{i}=0\right)$.

However, in a digital implementation, the time complexity would depend on the learning algorithm under consideration, dataset size and dimensionality, angular frequency $\omega$, and time constants $\tau_{i}$ for the phase updates.

\section{APPENDIX}

\section{A. Resonance in an LC tank}

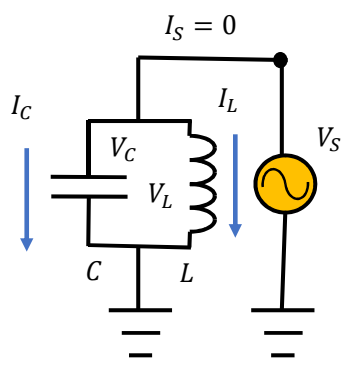

Consider the parallel LC tank circuit shown in Figure 12, with $V_{C}$ and $V_{L}$ being the voltages across the capacitor $C$ and inductor $L$ respectively. $I_{C}$ and $I_{L}$ denote the corresponding currents flowing through the elements. Thus, $V_{S}=V_{L}=V_{C}$ and $I_{S}=I_{L}+I_{C}$. Considering the LC tank to be driven by the in steady state:

voltage source $V_{S}$ at frequency $\omega$, we have the following condition

$$
I_{S}(\omega)=\frac{V_{S}(\omega)}{j \omega L}\left[1-\omega^{2} L C\right]
$$

Resonant condition of the circuit is achieved when

$$
\omega=\frac{1}{\sqrt{L C}} \Longrightarrow I_{S}(\omega)=0
$$

This implies that the apparent power $S_{N}=P_{N}+j Q_{N}=$ $V_{S} I_{S}^{*}+V_{L} I_{L}^{*}+V_{C} I_{C}^{*}$, where the active power $P_{N}=0$. Additionally at resonance, the reactive power $Q_{N}=Q_{C}+$ $Q_{L}=V_{L} I_{L}^{*}+V_{C} I_{C}^{*}=-\frac{j}{\omega L}|V(\omega)|^{2}+\frac{j}{\omega L}|V(\omega)|^{2}=0$. Here $Q_{C}$ and $Q_{L}$ are the reactive powers associated with the capacitance and inductance respectively.

\section{B. Mapping a generic optimization problem to the equivalent network model}

Let us consider an optimization problem defined over a probabilistic domain, given by the following generic form:

$$
\begin{array}{r}
\min _{\left\{x_{i}\right\}} \mathcal{H}\left(\left\{x_{i}\right\}\right) \\
\text { s.t. } \quad \sum_{i=1}^{N} x_{i}=1, \quad x_{i} \geq 0
\end{array}
$$

We can map the above to the electrical network-based model introduced in Section III by replacing $x_{i}=\left|V_{i}\right|^{2}+\left|I_{i}\right|^{2}$, which leads to the following problem in the $\left\{\left|V_{i}\right|^{2},\left|I_{i}\right|^{2}\right\}$ domain:

$$
\begin{array}{ll} 
& \min _{\substack{ \\
\\
\text { s.t. }}} \quad \sum_{i=1}^{N}\left(\left|I_{i}\right|\right\}\left(\left\{\left|V_{i}\right|,\left|I^{2}\right|\right\}\right) \\
&
\end{array}
$$


Note that the method also works for optimization problems defined over non-probabilistic domains, of the following form:

$$
\begin{array}{r}
\min _{\left\{x_{i}\right\}} \mathcal{H}\left(\left\{x_{i}\right\}\right) \\
\text { s.t. }\left|x_{i}\right| \leq 1, \quad x_{i} \in \mathbb{R} \quad \forall i=1, \ldots, N .
\end{array}
$$

This can be done by considering $x_{i}=x_{i}^{+}-x_{i}^{-} \forall i$, where both $x_{i}^{+}, x_{i}^{-} \geq 0$. Since by triangle inequality, $\left|x_{i}\right| \leq\left|x_{i}^{+}\right|+\left|x_{i}^{-}\right|$, enforcing $x_{i}^{+}+x_{i}^{-}=1 \forall i$ would automatically ensure $\left|x_{i}\right| \leq$ $1 \forall i$. Thus we have,

$$
\begin{aligned}
& \underset{\left\{x_{i}\right\}}{\operatorname{argmin}} \mathcal{H}\left(\left\{x_{i}\right\}\right) \equiv \underset{\left\{x_{i}^{+}, x_{i}^{-}\right\}}{\operatorname{argmin}} \mathcal{H}\left(\left\{x_{i}^{+}, x_{i}^{-}\right\}\right) \\
& \text {s.t. }\left|x_{i}\right| \leq 1, \quad x_{i} \in \mathbb{R} \quad \text { s.t. } \quad x_{i}^{+}+x_{i}^{-}=1, x_{i}^{+}, x_{i}^{-} \geq 0
\end{aligned}
$$

In this case, we replace $x_{i}^{+}=\left|V_{i}\right|^{2}, x_{i}^{-}=\left|I_{i}\right|^{2}$, and the equivalent problem in the $\left\{\left|V_{i}\right|^{2},\left|I_{i}\right|^{2}\right\}$ domain is thus as follows:

$$
\begin{array}{r}
\min _{\left\{\left|V_{i}\right|,\left|I_{i}\right|\right\}} \mathcal{H}\left(\left\{\left|V_{i}\right|,\left|I_{i}\right|\right\}\right) \\
\text { s.t. }\left|V_{i}\right|^{2}+\left|I_{i}\right|^{2}=1, \quad \forall i=1, \ldots, N,
\end{array}
$$

For example, the variables $\left\{x_{i}\right\}$ can represent the Lagrangian multipliers in the primal space of a support vector machine network, or the weights and biases of a generic neural network.

\section{Complex growth transform dynamical system (Table 2)}

Let us consider the optimization problem in Equation (34) again. We can use the Baum-Eagon inequality [40], [41] to converge to the optimal point of $\mathcal{H}$ in steady state, by using updates of the form

$$
x_{i} \leftarrow \frac{x_{i}\left(-\frac{\partial \mathcal{H}\left(\left\{x_{i}\right\}\right)}{\partial x_{i}}+\lambda\right)}{\sum_{k=1}^{N} x_{k}\left(-\frac{\partial \mathcal{H}\left(\left\{x_{k}\right\}\right)}{\partial x_{k}}+\lambda\right)},
$$

Here, $\mathcal{H}$ is assumed to be Lipschitz continuous [7] on the domain $D=\left\{x_{1}, \ldots, x_{N}: \sum_{i=1}^{N} x_{i}=1, x_{i} \geq 0 \forall i\right\} \subset \mathbb{R}_{+}^{N}$. The constant $\lambda \in \mathbb{R}_{+}$is chosen such that $\left|-\frac{\partial \mathcal{H}\left(\left\{x_{i}\right\}\right)}{\partial x_{i}}+\lambda\right|>$ $0, \forall i$.

We can solve the optimization problem given by Equation (10) by using the growth transforms discussed above. The outline of the proof is as follows: (1) We will start with a generic magnitude domain optimization problem without any phase regularizer and derive the form for the growth transform dynamical system which would converge to the optimal point asymptotically; (2) Next, we derive a complex domain counterpart of the above, again without phase constraints; (3) Finally, we derive the complex domain dynamical system by incorporating a phase regularizer in the objective function.

Since the time evolutions of $V_{i}$ and $I_{i}$ are symmetric because of the conservation constraints, for the rest of the section we will consider only the update equations for the voltages $V_{i}$, and similar results would also apply to the updates for $I_{i}$.
1) Condition 1: Considering $\beta=0$ in Equation (10) and $\mathcal{H}\left(\left\{\left|V_{i}\right|,\left|I_{i}\right|\right\}\right)$ to be Lipschitz continuous over the domain $D=\left\{\left|V_{i}\right|,\left|I_{i}\right|: \sum_{i=1}^{N}\left(\left|V_{i}\right|^{2}+\left|I_{i}\right|^{2}\right)=1\right\}$, we can use the growth transforms to arrive at the following discretetime update equations in terms of the voltage and current magnitudes :

$$
\left|V_{i, n}\right|^{2} \leftarrow g_{V_{i}, n-1}\left(\left\{\left|V_{i, n-1}\right|^{2},\left|I_{i, n-1}\right|^{2}\right\}\right),
$$

where

$$
\begin{gathered}
g_{V_{i}, n-1}\left(\left\{\left|V_{i, n-1}\right|^{2},\left|I_{i, n-1}\right|^{2}\right\}\right)=\frac{\left|V_{i, n-1}\right|^{2}}{\mu_{n-1}}\left(-\frac{\partial \mathcal{H}}{\partial\left|V_{i, n-1}\right|^{2}}+\lambda\right), \\
\mu_{n-1}=\sum_{k=1}^{N}\left(\left|V_{k, n-1}\right|^{2}\left[-\frac{\partial \mathcal{H}}{\partial\left|V_{k, n-1}\right|^{2}}+\lambda\right]\right. \\
\left.+\left|I_{k, n-1}\right|^{2}\left[-\frac{\partial \mathcal{H}}{\partial\left|I_{k, n-1}\right|^{2}}+\lambda\right]\right)
\end{gathered}
$$

and $\lambda \in \mathbb{R}_{+}$is chosen to ensure that $\left(-\frac{\partial \mathcal{H}}{\partial\left|V_{i}\right|^{2}}+\lambda\right)>0$ and $\left(-\frac{\partial \mathcal{H}}{\partial\left|I_{i}\right|^{2}}+\lambda\right)>0, \forall i$.

Writing $g_{V_{i}, n-1}=g_{V_{i}, n-1}\left(\left\{\left|V_{i, n-1}\right|^{2},\left|I_{i, n-1}\right|^{2}\right\}\right)$ for notational convenience and taking $g_{V_{i}, n-1}=\left|V_{i, n-1}\right|^{2} \sigma_{V_{i}, n-1}^{2}$, we get:

$$
\left|V_{i, n}\right|^{2} \leftarrow\left|V_{i, n-1}\right|^{2} \sigma_{V_{i}, n-1}^{2}
$$

2) Condition 2: Considering $\beta=0$ in Equation (10) and $V_{i}, I_{i} \in D^{C}=\left\{V_{i}, I_{i} \in \mathbb{C}: \sum_{i=1}^{N}\left(\left|V_{i}\right|^{2}+\left|I_{i}\right|^{2}\right)=1\right\}$, a time evolution of the form given below converges to limit cycles corresponding to the optimal point of a Lipschitz continuous objective function $\mathcal{H}\left(\left\{\left|V_{i}\right|,\left|I_{i}\right|\right\}\right)$ :

$$
\begin{aligned}
& V_{i, n} \leftarrow V_{i, n-1} \sigma_{V_{i}, n-1} e^{j \theta}, \\
& I_{i, n} \leftarrow I_{i, n-1} \sigma_{I_{i}, n-1} e^{j\left(\theta+\phi_{i}\right)}
\end{aligned}
$$

where $\sigma_{V_{i}}, \sigma_{I_{i}} \rightarrow 1 \quad \forall i=1, \ldots, N$, in steady state, $\theta$ is the instantaneous global phase difference of the system of phasors with respect to an absolute stationary reference frame, and $\phi_{i}$ is the instantaneous phase difference between $V_{i}$ and $I_{i}$.

Proof:

Since $\left|V_{i, n}\right|^{2}=V_{i, n} V_{i, n}^{*},\left|I_{i, n}\right|^{2}=I_{i, n} I_{i, n}^{*}$, the update equations can be rewritten as:

$\begin{aligned} V_{i, n} V_{i, n}^{*} & \leftarrow\left[V_{i, n-1} \sigma_{V_{i}, n-1} e^{j \theta}\right] \times\left[V_{i, n-1} \sigma_{V_{i}, n-1} e^{j \theta}\right]^{*} \\ I_{i, n} I_{i, n}^{*} & \leftarrow\left[I_{i, n-1} \sigma_{I_{i}, n-1} e^{j\left(\theta+\phi_{i}\right)}\right] \times\left[I_{i, n-1} \sigma_{I_{i}, n-1} e^{j\left(\theta+\phi_{i}\right)}\right]^{*}\end{aligned}$

where $\sigma_{V_{i}, n-1}$ is used to represent $\sigma_{V_{i}, n-1}\left(\left\{V_{i, n} V_{i, n}^{*}, I_{i, n} I_{i, n}^{*}\right\}\right)$ for ease of notation, and similarly for $\sigma_{I_{i}, n-1}$. Considering $\mathcal{H}\left(\left\{V_{i} V_{i}^{*}, I_{i} I_{i}^{*}\right\}\right)$ to be analytic in $D^{C}$ and applying Wirtinger's calculus [42], since

$$
\begin{aligned}
\frac{\partial \mathcal{H}}{\partial V_{i, n-1}} & =\frac{\partial \mathcal{H}}{\partial V_{i, n-1} V_{i, n-1}^{*}} \cdot\left(\frac{\partial V_{i, n-1} V_{i, n-1}^{*}}{\partial V_{i, n-1}}\right) \\
& =\frac{\partial \mathcal{H}}{\partial V_{i, n-1} V_{i, n-1}^{*}} \cdot V_{i, n-1}^{*},
\end{aligned}
$$


we have

$$
\sigma_{V_{i}, n-1}=\sqrt{\frac{1}{\eta_{i, n-1}}\left(-\frac{\partial \mathcal{H}}{\partial V_{i, n-1}}+\lambda V_{i, n-1}^{*}\right)}
$$

where

$$
\begin{gathered}
\eta_{i}=V_{i, n-1}^{*} \sum_{k=1}^{N}\left(V_{k, n-1}\left[-\frac{\partial \mathcal{H}}{\partial V_{k, n-1}}+\lambda V_{k, n-1}^{*}\right]\right. \\
\left.+I_{k, n-1}\left[-\frac{\partial \mathcal{H}}{\partial I_{k, n-1}}+\lambda I_{k, n-1}^{*}\right]\right)
\end{gathered}
$$

The discrete time update equations for $V_{i, n}$ is thus given by:

$$
V_{i, n} \leftarrow V_{i, n-1} \sigma_{V_{i}, n-1} e^{j \theta}
$$

Similar expressions can also be derived for the current phasors.

Lemma: A continuous-time variant of the model given by Equation (44) is given by:

$$
\frac{\partial V_{i}(t)}{\partial t}=j \omega \sigma_{V_{i}}(t) V_{i}(t)-\Delta \sigma_{V_{i}}(t) V_{i}(t) .
$$

Proof: The difference equations for the voltage phasors are given by:

$V_{i, n}-V_{i, n-1} \leftarrow V_{i, n-1} \sigma_{V_{i}, n-1}\left[e^{j \theta}-1\right]+V_{i, n-1}\left[\sigma_{V_{i}, n-1}-1\right]$

which can be rewritten as:

$V_{i, n}-V_{i, n-1} \leftarrow V_{i, n-1} \sigma_{V_{i}, n-1}\left[\mathrm{e}^{j \omega \Delta t}-1\right]-V_{i, n-1} \Delta \sigma_{V_{i}, n-1}$,

where $\Delta \sigma_{V_{i}, n-1}=1-\sigma_{V_{i}, n-1}$, and $\theta=\omega \Delta t$, where $\omega$ is the common oscillation frequency of all the phasors, and $\Delta t$ is the time increment. In the limiting case, when $\Delta t \rightarrow 0$, this reduces to the following continuous time differential equation for the complex variable $V_{i}(t)$ :

$$
\frac{\partial V_{i}(t)}{\partial t}=j \omega \sigma_{V_{i}}(t) V_{i}(t)-\Delta \sigma_{V_{i}}(t) V_{i}(t),
$$

In the steady state, since $H$ is Lipschitz continuous in $D$, $\sigma_{V_{i}}(t) \stackrel{t \rightarrow \infty}{\longrightarrow} 1$, the dynamical system above thus reduces to:

$$
\frac{\partial V_{i}(t)}{\partial t}=j \omega V_{i}(t) .
$$

which implies that the steady state response of the complex variable $V_{i}(t)$ corresponds to steady oscillations with a constant angular frequency of $\omega$.

The difference equations in terms of the nodal currents can be similarly written as:

$$
\begin{array}{r}
I_{i, n}-I_{i, n-1} \leftarrow I_{i, n-1} \sigma_{I_{i}, n-1}\left[\mathrm{e}^{j\left(\omega+\omega_{\phi_{i}}\right) \Delta t}-1\right] \\
-I_{i, n-1} \Delta \sigma_{I_{i}, n-1},
\end{array}
$$

where $\omega_{\phi_{i}}=\frac{d \phi_{i}}{d t}$. The equivalent continuous domain differential equation is then given by:

$$
\frac{\partial I_{i}(t)}{\partial t}=j\left(\omega+\omega_{\phi_{i}}\right) \sigma_{I_{i}}(t) I_{i}(t)-\Delta \sigma_{I_{i}}(t) I_{i}(t) .
$$

3) Condition 3: Considering $\beta \neq 0$ in Equation (10), additional phase constraints can be incorporated in the dynamical system represented by using the update rules in Equations [15)-(18). In steady state, for $\left|V_{i}\right|^{2}\left|I_{i}\right|^{2} \neq 0$, the system settles to $\phi_{i}= \pm \pi / 2 \forall i$. Additionally, for sufficiently small values of $\beta$ (or if $\beta$ is slowly increased during the optimization process), the system converges to the optimal point of the modified objective function $\mathcal{H}\left(\left\{\left|V_{i}\right|,\left|I_{i}\right|\right\}\right)$.

Proof: Since $\mathcal{L}\left(\left\{\left|V_{i}\right|,\left|I_{i}\right|, \phi_{i}\right\}\right)$ is Lipschitz continuous in both $\left|V_{i}\right|^{2}$ and $\left|I_{i}\right|^{2}$, the same form of the update equations proved in Lemma 2 can be applied. For arriving at the updates for the phase angles $\phi_{i}$, we will use a similar approach as shown in Equation (37). We can split $\phi_{i}$ as $\phi_{i}=\phi_{i}^{+}-\phi_{i}^{-}, \phi_{i}^{+}, \phi_{i}^{-}>0$, which implies that $\phi_{i}^{+}+\phi_{i}^{-}=\pi$. We can then apply the growth transform dynamical system [7] to get

$$
\text { and } \tau_{i} \omega_{\phi_{i}}+\phi_{i}(t)=g_{\phi_{i}}(t) \text {, }
$$

where $\omega_{\phi_{i}}=\frac{d \phi_{i}(t)}{d t}$ and

$$
g_{\phi_{i}}=\pi \frac{\phi_{i}^{+}\left(-\frac{\partial \mathcal{L}}{\partial \phi_{i}^{+}}+\lambda\right)-\phi_{i}^{-}\left(-\frac{\partial \mathcal{L}}{\partial \phi_{i}^{-}}+\lambda\right)}{\phi_{i}^{+}\left(-\frac{\partial \mathcal{L}}{\partial \phi_{i}^{+}}+\lambda\right)+\phi_{i}^{-}\left(-\frac{\partial \mathcal{L}}{\partial \phi_{i}^{-}}+\lambda\right)}
$$

Since $\frac{\partial \mathcal{L}}{\partial \phi_{i}}=\frac{\partial \mathcal{L}}{\partial \phi_{i}^{+}}=-\frac{\partial \mathcal{L}}{\partial \phi_{i}^{-}}$, the above can be simplified to $g_{\phi_{i}}=\pi \frac{\lambda \phi_{i}-\pi \frac{\partial \mathcal{L}}{\partial \phi_{i}}}{-\phi_{i} \frac{\partial \mathcal{L}}{\partial \phi_{i}}+\lambda \pi}$ This implies that the voltage and current phasors corresponding to the $i$-th node in the network may be phase shifted by an additional amount $\phi_{i}$ with respect to the absolute reference.

Since for optimality, $\phi_{i}= \pm \pi / 2$ for $\left|V_{i}\right|^{2}\left|I_{i}\right|^{2} \neq 0$ in the steady state, the net energy dissipation in steady state is zero, i.e., $\sum_{n=1}^{N}\left|V_{i}\right|^{2}\left|I_{i}\right|^{2} \cos ^{2} \phi_{i}=0$. Also, in the steady state, for sufficiently small values of the hyperparameter $\beta$,

$\underset{\left\{\left|V_{i}\right|,\left|I_{i}\right|, \phi_{i}\right\}}{\operatorname{minimize}} \mathcal{L}\left(\left\{\left|V_{i}\right|,\left|I_{i}\right|, \phi_{i}\right\}\right)=\underset{\left\{\left|V_{i}\right|,\left|I_{i}\right|\right\}}{\operatorname{minimize}} \mathcal{H}\left(\left\{\left|V_{i}\right|,\left|I_{i}\right|\right\}\right)$,

which implies that the system reaches the optimal solution with zero active power in the post-learning stage.

\section{REFERENCES}

[1] Y. LeCun, S. Chopra, R. Hadsell, M. Ranzato, and F. Huang, "A tutorial on energy-based learning," Predicting Structured Data, vol. 1, no. 0, 2006.

[2] J. J. Hopfield and D. W. Tank, "Neural computation of decisions in optimization problems," Biological Cybernetics, vol. 52, no. 3, pp. 141$152,1985$.

[3] J. Edminister, Theory and problems of electric circuits. Schaum, 1965

[4] M. E. V. Valkenburg, Network analysis. Prentice-Hall, 1964.

[5] J. Dixon, L. Moran, J. Rodriguez, and R. Domke, "Reactive power compensation technologies: State-of-the-art review," Proceedings of the IEEE, vol. 93, no. 12, pp. 2144-2164, 2005.

[6] W. Hofmann, J. Schlabbach, and W. Just, Reactive power compensation: a practical guide. John Wiley \& Sons, 2012. 
[7] O. Chatterjee and S. Chakrabartty, "Decentralized global optimization based on a growth transform dynamical system model," IEEE Transactions on Neural Networks and Learning Systems, vol. 29, no. 12, pp. 6052-6061, 2018

[8] B. Schölkopf, R. C. Williamson, A. J. Smola, J. Shawe-Taylor, and J. C. Platt, "Support vector method for novelty detection," in Advances in Neural Information Processing Systems, pp. 582-588, 2000.

[9] S. H. Strogatz, "From kuramoto to crawford: exploring the onset of synchronization in populations of coupled oscillators," Physica D: Nonlinear Phenomena, vol. 143, no. 1-4, pp. 1-20, 2000.

[10] R. Feynman, "The character of physical law," 2017.

[11] Y.-L. Boureau, S. Chopra, Y. LeCun, et al., "A unified energy-based framework for unsupervised learning," in Artificial Intelligence and Statistics, pp. 371-379, 2007.

[12] G. E. Hinton and T. J. Sejnowski, "Optimal perceptual inference," in Proceedings of the IEEE conference on Computer Vision and Pattern Recognition, pp. 448-453, 1983.

[13] R. Salakhutdinov, A. Mnih, and G. Hinton, "Restricted boltzmann machines for collaborative filtering," in Proceedings of the 24th international conference on Machine learning, pp. 791-798, ACM, 2007.

[14] B. Scellier and Y. Bengio, "Equilibrium propagation: Bridging the gap between energy-based models and backpropagation," Frontiers in Computational Neuroscience, vol. 11, p. 24, 2017.

[15] J. Ngiam, Z. Chen, P. W. Koh, and A. Y. Ng, "Learning deep energy models," in Proceedings of the 28 th International Conference on Machine Learning, 2011.

[16] S. Zhai, Y. Cheng, W. Lu, and Z. Zhang, "Deep structured energy based models for anomaly detection," in International Conference on Machine Learning, pp. 1100-1109, 2016.

[17] T. Haarnoja, H. Tang, P. Abbeel, and S. Levine, "Reinforcement learning with deep energy-based policies," in Proceedings of the 34th International Conference on Machine Learning-Volume 70, pp. 1352-1361, JMLR. org, 2017.

[18] J. Lawson, G. Tucker, B. Dai, and R. Ranganath, "Energy-inspired models: Learning with sampler-induced distributions," in Advances in Neural Information Processing Systems, pp. 8499-8511, 2019.

[19] A. Hirose, Complex-valued neural networks: theories and applications, vol. 5. World Scientific, 2003.

[20] C. Trabelsi, O. Bilaniuk, Y. Zhang, D. Serdyuk, S. Subramanian, J. F. Santos, S. Mehri, N. Rostamzadeh, Y. Bengio, and C. J. Pal, "Deep complex networks," arXiv preprint arXiv:1705.09792 2017.

[21] C. J. Gaudet and A. S. Maida, "Deep quaternion networks," in 2018 International Joint Conference on Neural Networks (IJCNN), pp. 1-8, IEEE, 2018.

[22] N. Guberman, "On complex valued convolutional neural networks," arXiv preprint arXiv:1602.09046 2016.

[23] P. Bouboulis, S. Theodoridis, C. Mavroforakis, and L. EvaggelatouDalla, "Complex support vector machines for regression and quaternary classification," IEEE Transactions on Neural Networks and Learning Systems, vol. 26, no. 6, pp. 1260-1274, 2014.

[24] J. Bruna, S. Chintala, Y. LeCun, S. Piantino, A. Szlam, and M. Tygert, "A mathematical motivation for complex-valued convolutional networks," arXiv preprint arXiv:1503.03438 2015.

[25] T. Nitta, "Orthogonality of decision boundaries in complex-valued neural networks," Neural Computation, vol. 16, no. 1, pp. 73-97, 2004.

[26] T. Nakashika, S. Takaki, and J. Yamagishi, "Complex-valued restricted boltzmann machine for direct learning of frequency spectra.," in INTERSPEECH, pp. 4021-4025, 2017.

[27] B. D. Tellegen, "A general network theorem, with applications," Philips Res Rep, vol. 7, pp. 256-269, 1952.

[28] T. Hastie, R. Tibshirani, J. Friedman, and J. Franklin, "The elements of statistical learning: data mining, inference and prediction," The Mathematical Intelligencer, vol. 27, no. 2, pp. 83-85, 2005.

[29] K. P. Murphy, Machine learning: a probabilistic perspective. MIT press, 2012.

[30] Z. Ghahramani, "Probabilistic machine learning and artificial intelligence," Nature, vol. 521, no. 7553, p. 452, 2015.

[31] S. Chakrabartty and G. Cauwenberghs, "Gini support vector machine: Quadratic entropy based robust multi-class probability regression," Journal of Machine Learning Research, vol. 8, no. Apr, pp. 813-839, 2007.

[32] D. M. Tax and R. P. Duin, "Support vector domain description," Pattern Recognition Letters, vol. 20, no. 11-13, pp. 1191-1199, 1999.

[33] A. Gangopadhyay, O. Chatterjee, and S. Chakrabartty, "Extended polynomial growth transforms for design and training of generalized support vector machines," IEEE Transactions on Neural Networks and Learning Systems, vol. 29, no. 5, pp. 1961-1974, 2017.
[34] D. Dua and C. Graff, "UCI machine learning repository." http://archive. ics.uci.edu/ml 2017.

[35] S. Rayana, "ODDS library.” http://odds.cs.stonybrook.edu 2016.

[36] A. Raychowdhury, A. Parihar, G. H. Smith, V. Narayanan, G. Csaba, M. Jerry, W. Porod, and S. Datta, "Computing with networks of oscillatory dynamical systems," Proceedings of the IEEE, vol. 107, no. 1, pp. 73-89, 2019.

[37] J. Torrejon, M. Riou, F. A. Araujo, S. Tsunegi, G. Khalsa, D. Querlioz, P. Bortolotti, V. Cros, K. Yakushiji, A. Fukushima, et al., "Neuromorphic computing with nanoscale spintronic oscillators," Nature, vol. 547, no. 7664, p. 428, 2017.

[38] M. A. Nielsen and I. L. Chuang, Quantum computation and quantum information. Cambridge university press, 2010.

[39] A. Gangopadhyay, O. Chatterjee, and S. Chakrabartty, "Continuoustime optimization using sub-threshold current-mode growth transform circuits," in 2018 IEEE 61st International Midwest Symposium on Circuits and Systems (MWSCAS), pp. 246-249, IEEE, 2018.

[40] L. E. Baum and G. Sell, "Growth transformations for functions on manifolds," Pacific Journal of Mathematics, vol. 27, no. 2, pp. 211227, 1968.

[41] P. Gopalakrishnan, D. Kanevsky, A. Nadas, and D. Nahamoo, "A generalization of the baum algorithm to rational objective functions," in International Conference on Acoustics, Speech, and Signal Processing, pp. 631-634, IEEE, 1989.

[42] M. F. Amin, M. I. Amin, A. Y. H. Al-Nuaimi, and K. Murase, "Wirtinger calculus based gradient descent and levenberg-marquardt learning algorithms in complex-valued neural networks," in International Conference on Neural Information Processing, pp. 550-559, Springer, 2011. 